\title{
The X-ray spectral properties of the AGN population in the XMM-Newton bright serendipitous survey ${ }^{\star}$
}

\author{
A. Corral $^{1}$, R. Della Ceca ${ }^{1}$, A. Caccianiga ${ }^{1}$, P. Severgnini ${ }^{1}$, H. Brunner ${ }^{2}$, F. J. Carrera ${ }^{3}$, \\ M. J. Page ${ }^{4}$, and A. D. Schwope ${ }^{5}$
}

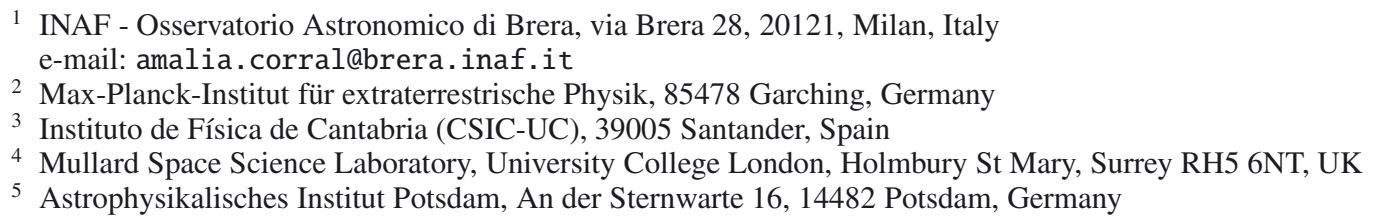

Received 17 June 2010 / Accepted 9 March 2011

\begin{abstract}
Context. X-ray surveys are a key instrument in the study of active galactic nuclei (AGN). Thanks to their penetrating ability, X-rays are able to map the innermost regions close to the central super massive black hole (SMBH) as well as to detect and characterize its emission up to high redshift.

Aims. We present here a detailed X-ray spectral analysis of the AGN belonging to the XMM-Newton bright survey (XBS). The XBS is composed of two flux-limited samples selected in the complementary $0.5-4.5$ and $4.5-7.5 \mathrm{keV}$ energy bands and comprising more than 300 AGN up to redshift $\sim 2.4$.

Methods. We performed an X-ray analysis following two different approaches: by analyzing individually each AGN X-ray spectrum and by constructing average spectra for different AGN types.

Results. From the individual analysis, we find that there seems to be an anti correlation between the spectral index and the sources' hard X-ray luminosity, such that the average photon index for the higher luminosity sources $\left(>10^{44} \mathrm{erg} \mathrm{s}^{-1}\right)$ is significantly $(>2 \sigma)$ flatter than the average for the lower luminosity sources. We also find that the intrinsic column density distribution agrees with AGN unified schemes, although a number of exceptions are found (3\% of the whole sample), which are much more common among optically classified type 2 AGN. We also find that the so-called "soft-excess", apart from the intrinsic absorption, constitutes the principal deviation from a power-law shape in AGN X-ray spectra and it clearly displays different characteristics, and likely a different origin, for unabsorbed and absorbed AGN. Regarding the shape of the average spectra, we find that it is best reproduced by a combination of an unabsorbed (absorbed) power law, a narrow Fe K $\alpha$ emission line and a small (large) amount of reflection for unabsorbed (absorbed) sources. We do not significantly detect any relativistic contribution to the line emission and we compute an upper limit for its equivalent width $(E W)$ of $230 \mathrm{eV}$ at the $3 \sigma$ confidence level. Finally, by dividing the type 1 AGN sample into high- and low-luminosity sources, we marginally detect a decrease in the narrow $\mathrm{Fe} \mathrm{K} \alpha$ line $E W$ and in the amount of reflection as the luminosity increases, the "so-called" Iwasawa-Taniguchi effect.
\end{abstract}

Key words. X-rays: general - X-rays: diffuse background - surveys - galaxies: active

\section{Introduction}

Recent deep X-rays surveys carried out by XMM-Newton and Chandra have resolved most of the cosmic X-ray background (CXB) into discrete sources up to energies $\sim 10 \mathrm{keV}$ (although the resolved fraction decreases with energy; Worsley et al. 2005; Hickox \& Markevitch 2006). The large majority of the sources that compose the CXB are active galactic nuclei (AGN), and CXB synthesis models make use of template AGN spectra to reproduce its shape following the AGN unified model (Antonucci 1993). The unified model, in its simplest version, states that the differences between the different observed AGN types are due to an orientation effect, i.e., as the inclination angle to the observer increases, the torus surrounding the central super massive black hole (SMBH) intercepts more nuclear emission. The CXB

\footnotetext{
* Appendix and Tables 2, 3 are available in electronic form at http://wWw . aanda.org
}

is then reproduced by a mixture of AGN spectra with different amounts of absorption.

However, there are still many unresolved questions regarding our knowledge about AGN. For example, the predicted fraction of heavily absorbed AGN (Compton-thick AGN) obtained from CXB synthesis models can vary from $30 \%$ to $9 \%$ between different works (Gilli et al. 2007; Treister et al. 2009). Besides, a small number of AGN that seem not to follow the unified scheme are usually found in X-rays surveys, i.e., their optical characteristics do not match their observed X-ray properties (Panessa \& Bassani 2002; Akylas et al. 2004; Caccianiga et al. 2004; Cappi et al. 2006; Mateos et al. 2005a,b, 2010). The evolution of these properties through cosmic time and the possible correlation between X-ray emission and source properties, like the bolometric luminosity or SMBH mass, are also a matter of debate.

The frequency and properties of some individual characteristics are also still unknown, such as the $\mathrm{Fe} \mathrm{K} \alpha$ emission line. This 
emission line is the most commonly observed line in AGN X-ray spectra, but its detailed study is strongly limited by the data quality and therefore, to sources in the local Universe (Nandra et al. 2007). To study its characteristics up to high redshifts, X-ray spectra have to be stacked to improve the signal-to-noise ratio (SNR) (Corral et al. 2008; Streblyanska et al. 2005; Brusa et al. 2005). Another intriguing AGN feature is the soft-excess emission in type 1 AGN, whose origin is still unclear. Possible suggested explanations go from continuum emission (Ross et al. 1992; Shimura \& Takahara 1993; Kawaguchi et al. 2001) to atomic processes (Crummy et al. 2006; Middleton et al. 2007).

Deep and medium surveys often lack good quality X-ray and multi-wavelength data, which limits the results, while samples composed by high-quality data are usually not well-defined flux-limited samples, which limits the applicability of the results. Given all that, well-defined X-ray samples, that contain both a significant number of reliable identifications and good enough X-ray data quality are the key to test the current hypotheses and to link the nearby and distant universe. We present here a detailed X-ray spectral analysis of the AGN within the XBS sample, which is composed of two flux-limited samples that are almost completely identified (identification rate $~ 95 \%$ ) and containing more than 300 AGN. Given the availability of both reliable optical spectroscopic identifications and good quality X-ray spectral data, this sample is the perfect laboratory to test AGN models and to better constrain the AGN properties and their evolution.

We assume the cosmological model $H_{O}=65 \mathrm{~km} \mathrm{~s}^{-1} \mathrm{Mpc}^{-1}$, $\Omega_{\lambda}=0.7$ and $\Omega_{\mathrm{M}}=0.3$ throughout this paper. Reported errors are at $90 \%$ confidence level unless stated otherwise.

\section{The XBS AGN sample}

The sample of 305 AGN discussed here (XBS AGN sample hereafter) has been extracted from the XMM-Newton bright serendipitous survey ${ }^{1}$.

The XBS consists of two flux-limited serendipitous (i.e. the targets of the XMM-Newton pointings were excluded) samples of X-ray selected sources at high galactic latitude $(|b|>$ $20^{\circ}$ ): the XMM bright serendipitous survey sample (BSS, 389 sources) and the XMM hard bright serendipitous survey sample (HBSS, 67 sources, with 56 sources in common with the BSS sample) having an EPIC MOS2 count rate limit, corrected for vignetting, of $10^{-2} \mathrm{cts} / \mathrm{s}$ and $2 \times 10^{-3} \mathrm{cts} / \mathrm{s}$ in the $0.5-4.5 \mathrm{keV}$ and $4.5-7.5 \mathrm{keV}$ energy bands, respectively; the flux limit is $\sim 7 \times 10^{-14} \mathrm{erg} \mathrm{cm}^{-2} \mathrm{~s}^{-1}$ in both energy selection bands.

The details on the XMM-Newton fields selection strategy and the source selection criteria of the XMM BSS and HBSS samples are discussed in Della Ceca et al. (2004), while a description of the optical data and analysis, of the optical classification scheme and the optical properties of the extragalactic sources identified so far is presented in Caccianiga et al. (2007, 2008). The optical and X-ray properties of the Galactic population are discussed in López-Santiago et al. (2007). Previous X-rays spectral analyses of parts of the XBS sample have already been reported in previous works. In Caccianiga et al. (2004), the X-ray

\footnotetext{
1 The XMM-Newton Bright Serendipitous Survey is one of the research programs conducted by the XMM-Newton Survey Science Center (SSC, see http://xmmssc-www.star.le.ac.uk) a consortium of 10 international institutions, appointed by ESA to help the SOC in developing the software analysis system, to pipeline process all the $X M M$-Newton data, and to exploit the XMM serendipitous detections. The Osservatorio Astronomico di Brera is one of the Consortium Institutes.
}

spectral analysis of a subsample extracted form the HBSS sample is reported. Galbiati et al. (2005) performed an analysis of the radio-loud AGN within the XBS. Severgnini et al. (2003) unveiled the AGN-nature of three sources previously considered as normal galaxies. Finally, Della Ceca et al. (2008) presented the cosmological properties of the HBSS AGN sample.

\subsection{AGN classification}

The current classification breakdown of the XBS sample, which relies on dedicated optical spectroscopy, is as follows: 305 AGN (including 5 BL Lacs), 8 clusters of galaxies ${ }^{2}, 2$ normal galaxies and $58 \mathrm{X}$-ray emitting stars, see Table 1 for a detailed summary. For 25 out of the 305 AGN that composed our sample, redshift and classification are reported here for the first time. These new identifications are marked in boldface in Table 3 (Cols. 2 and 3). The XBS AGN sample contains 35 sources that are optically classified as elusive AGN, i.e., sources for which a classification cannot be derived solely from our optical spectroscopy, although the redshift can be measured. These are sources that are characterized by a significant/dominant contamination of starlight from the host galaxy in the optical spectrum (Caccianiga et al. 2007). Even if the presence of an AGN in these sources is somehow suggested by the detection of a broad or strong emission line, the "dilution" caused by the host galaxy is critical because it avoids the quantification of the optical absorption that is necessary to classify a source as a type 1 or type 2 AGN. For these sources, the type $1 / 2$ classification is assigned as a function of the absence/presence of a significant amount of intrinsic absorption in their X-ray spectra. There is one case however, XBSJ012654.3+191246, in which a type $1 / 2$ classification cannot be inferred from either the optical or the X-ray data, and accordingly this source is classified as an AGN of uncertain type. At the time of writing, $27 \mathrm{X}$-ray sources belonging to the BSS sample are still unidentified. Out of these 27, two also belong to the HBSS sample, which results in a level of identification of $93 \%$ and $97 \%$ for the BSS and the HBSS samples, respectively.

\subsection{X-ray data}

The XBS source sample was defined using only the data from the MOS2 detector. However, to increase the statistics, the data from the MOS1 and the pn detectors were considered when available and were used for our spectral analysis.

In Table 2 we report the data used for the X-ray spectral analysis of each source: Source name; XMM-Newton observation ID; XMM-Newton filter for each detector; the values of Galactic column densities toward the used XMM-Newton pointings; resulting exposure time for each detector after removing highbackground intervals; total counts for all available detectors in the $0.3-10 \mathrm{keV}$ band, and corresponding sample. To increase the number of counts for the lowest quality spectra, we searched the XMM-Newton archive for additional observations and selected those with the longest MOS2 exposure times; we preferred not to combine different observation data sets to minimize possible problems related to source variability. As a result, some of the data sets used in this analysis are different from the ones used in Della Ceca et al. (2004) for the definition of the sample.

\footnotetext{
2 The sample of cluster of galaxies is neither statistically complete nor representative of the cluster population because the source detection algorithm used in the construction of the sample is optimized for pointlike sources.
} 
Table 1. XBS classification summary.

\begin{tabular}{lcccccccc}
\hline \hline Sample & Type 1 AGN & Type 2 AGN & BL Lacs & Stars & Clusters & Galaxies & Unidentified & AGN uncertain type \\
\hline BSS & $269(41)$ & $19(10)$ & 5 & $58(2)$ & $8(1)$ & 2 & $27(2)$ & 1 \\
HBSS & 42 & 20 & $\ldots$ & 2 & 1 & $\ldots$ & 2 & $\ldots$ \\
\hline Total & 270 & 29 & 5 & 58 & 8 & 2 & 27 & 1 \\
\hline
\end{tabular}

Notes. The numbers between parenthesis for the BSS sample correspond to the number of sources in common with the HBSS sample.

The XMM-Newton data were cleaned and processed with the $X M M-N e w t o n$ Science Analysis Software (SAS) and were analyzed with standard software packages (Ftools; Xspec, Arnaud 1996). Event files produced from the pipeline were filtered from high-background time intervals and only events corresponding to pattern 0-12 for MOS and 0-4 for pn were used. All spectra were accumulated from a circular extraction region with a radius of $\sim 20^{\prime \prime}-30^{\prime \prime}$, depending on the source off-axis distance. Background counts were accumulated in nearby circular source free regions which an area usually about a factor $\sim 4$ larger than the one used to extract the source counts. To improve the statistics, the MOS1 and MOS2 spectra obtained with the same filters were combined a posteriori by using the FTOOLS task mathpha. The X-ray spectra usually cover the $0.3-10 \mathrm{keV}$ energy range; the total (MOS1+MOS2+pn) counts range from $\sim 100$ to $\sim 10^{4}$, as can be seen in Fig. 1.

The ancillary response matrix and the detector response matrix were created by the XMM-SAS task arfgen and rmfgen at each source position in the EPIC detectors. For the MOS1 and MOS2 detectors, and provided that the observations were carried out by using the same filter, ancillary and detector response matrices for each source were combined by using addrmf and addarf.

\section{X-ray spectral analysis}

The availability of good XMM-Newton data for the sources in the XBS sample, which spans the energy range between $\sim 0.3$ and $\sim 10 \mathrm{keV}$, allow us to perform a reliable X-ray spectral analysis for almost every AGN studied here. For 111 AGN an $\mathrm{X}$-ray spectral analysis was already reported and discussed in Severgnini et al. (2003), Caccianiga et al. (2004, 2007), Galbiati et al. (2005) or in Della Ceca et al. (2008); for the remaining AGN the main X-ray spectral properties and parameters are discussed in detail here for the first time. Note, however, that there could be small differences in the best-fit model and parameters already published and the ones presented here owing to the different XMM-Newton observations used and/or our different way of defining the best-fit model for each source.

We grouped the spectra in bins containing at least 10 to 30 (depending on the spectral quality) source+background counts to use the $\chi^{2}$ minimization technique. We fitted pn and MOS spectra simultaneously in the $0.3-10 \mathrm{keV}$ band with Xspec version 12.5.0. We tied together all pn and MOS parameters except for a relative normalization, which accounts for the differences between pn and MOS flux calibrations. In the following, derived fluxes and luminosities refer to the MOS2 calibration.

To ensure a spectral analysis as uniform as possible, we defined a threshold of $10 \%$ for the null hypothesis probability to distinguish between an acceptable and an unacceptable fit, i.e., we consider as our best-fit model the simplest model for which the probability is $>10 \%$.
As a starting point for the spectral modeling we first considered a simple absorbed power-law model that takes into account both the Galactic hydrogen column density along the line of sight (from Dickey \& Lockman 1990) and a possible intrinsic absorption at the source redshift (Xspec model: wabs*zwabs*zpo). In the X-ray spectral modeling we made use of the redshifts obtained from the optical spectroscopy.

The results for this simple fit are shown in Table 3 along with the corresponding Galactic de-absorbed flux and intrinsic luminosity in the standard hard $(2-10 \mathrm{keV})$ energy band. In some cases, the spectral quality does not allow us to constrain the power-law photon index $(\Gamma)$ and the intrinsic absorption at the same time. In other cases, the resulting photon index is $\sim 1$, much lower than the typical values for unabsorbed $\mathrm{AGN}^{3}$. In those cases, we fixed $\Gamma$ to 1.9 , a common value for unabsorbed AGN (Caccianiga et al. 2004; Mateos et al. 2005a,b; Galbiati et al. 2005; Tozzi et al. 2006; Mateos et al. 2010). If there was no intrinsic absorption detected, an upper limit, at $90 \%$ confidence level, is given. The simple absorbed power-law model gives a good fit for 263 sources, but seems to fail in reproducing the spectral shape for 41 sources, marked with a ${ }^{p}$ in Table 3. All $\mathrm{X}$-ray spectra corresponding to sources classified as BL Lacs are well fitted by the simple power-law model.

For the 41 sources that are not well fitted by an absorbed power law, we tried several additional components to the absorbed power law model. We accept any of these additional components if the improvement of the fit was larger than $95 \%$ as measured by an F-test. These additional components are

- Leaky absorbed power-law: an additional unabsorbed power-law component, with the same photon index as the direct one, representing scattered emission into our line of sight (Xspec model: wabs (zwabs*zpo+zpo)). This model can also account for partially covered emission.

- Ionized absorption: (Xspec model: wabs*zwabs*absori * zpo, Magdziarz \& Zdziarski 1995), since signatures of absorption from partially ionized gas have been found to be a quite common characteristic in the spectra of Seyfert galaxies.

- Reflected component: to account for a spectral hardening or change of curvature at high energies because of Compton reflection from neutral material (Xspec model: wabs (zwabs*zpo+pexrav), Magdziarz \& Zdziarski 1995). We fixed the inclination angle to $\sim 60 \mathrm{deg}$, an average value

\footnotetext{
${ }^{3}$ An alternative possibility is that these sources with an observed flat spectrum are Compton-thick AGN (i.e. sources with $N_{\mathrm{H}}>10^{24} \mathrm{~cm}^{-2}$ ) in which all the direct emission is suppressed and only reflected emission is observed (in the $2-10 \mathrm{keV}$ band). This effect, combined with the low statistics, may mimic a flat spectrum. However, this hypothesis does not seem to be valid in our sources because in all cases we find a significant amount of absorption (but not in the Compton-thick regime) even when leaving the photon index as a free parameter $\left(N_{\mathrm{H}}\right.$ from $3.5 \times 10^{21}$ to $2 \times 10^{23} \mathrm{~cm}^{-2}$ ). We conclude that the best explanation for the sources with a very flat spectral index is the combination of (mild) absorption and the low statistics
} 

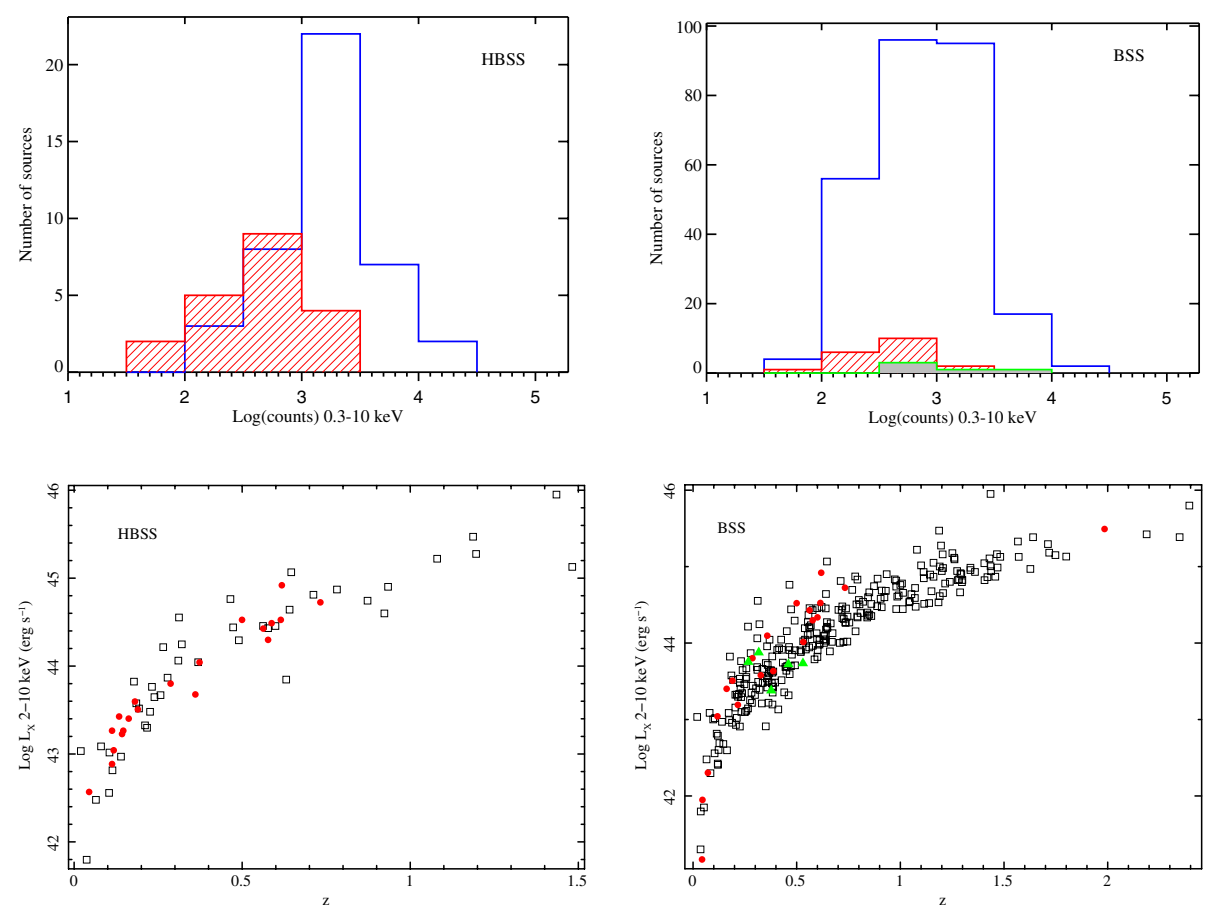

Fig. 1. Top panels: counts distribution for the HBSS (left) and the BSS (right) samples. Empty histograms correspond to type 1 AGN and dashed histograms to type 2 AGN. Filled histogram for the BSS corresponds to BL Lacs. Bottom panels: X-ray intrinsic luminosity versus redshift for the HBSS (left) and the BSS (right). Squares and circles correspond to sources classified as type 1 and type 2 AGN, respectively. Triangles on the BSS sample represent BL Lacs.

for Seyfert galaxies, because the spectral quality does not allow us to constrain it and the reflection factor $R(R=\Omega / 2 \pi)$ at the same time.

- Thermal component to account for soft emission lines that could arise from ionized material far from the central source, like the narrow-line region (NLR) (Xspec model: wabs (zwabs*zpo+mekal), Mewe et al. 1986; Liedahl et al. 1995). Although the NLR is likely photoionized, we can approximate the resulting spectral shape by using this collisional model given the spectral resolution for the EPIC cameras.

- Lines and edges (zgauss, zegde) to model emission lines (such as the Fe $\mathrm{K} \alpha$ emission line, the most commonly observed one in AGN X-ray spectra) and absorption edges. Energies were left free to vary.

- A phenomenological black body model (Xspec model: wabs (zwabs*zpo+zbb) to account for featureless softexcess emission.

If different additional components significantly improved the fit, we selected the model that was more physically plausible and/or gave better residuals. These cases, $28 \mathrm{AGN}$, are discussed in more detail in the Appendix. There are also two cases in which more than one additional component are required to obtain an acceptable fit: in both cases, one of the required additional components turned out to be an emission line. A summary of the models required during the spectral fit is shown in Table 4, while the results for the additional component fits are presented in Tables 6 to 11. All models that significantly improve the simple absorbed power-law fit are shown for each source in Tables 6 to 11. The model we considered as the "best-fit" for each source is marked in boldface in those tables and its parameters are the ones we consider in the interpretation of the results.

In 11 cases still no acceptable fit was found. This can be simply due to a statistical effect given the $10 \%$ probability limit imposed to consider a fit as acceptable. Roughly, $10 \%$ of the sources that actually display a power-law shape are expected
Table 4. X-ray spectral fit.

\begin{tabular}{lccc}
\hline \hline Model & Type 1 AGN & Type 2 AGN & BL Lacs \\
\hline SPL & 243 & 24 & 5 \\
Leaky & 1 & 3 & $\ldots$ \\
PL+T & $\ldots$ & 2 & $\ldots$ \\
PL+BB & 13 & $\ldots$ & $\ldots$ \\
WAPL & 5 & $\ldots$ & $\ldots$ \\
PL+R & 4 & $\ldots$ & $\ldots$ \\
PL+E & 3 & $\ldots$ & $\ldots$ \\
Leaky+L & 1 & 1 & $\ldots$ \\
\hline
\end{tabular}

Notes. SPL: absorbed power law; Leaky: absorbed plus unabsorbed power law. PL+T: absorbed power law plus thermal component; PL+BB: absorbed power law plus black body component; WAPL: ionized absorbed power law; PL+R: absorbed power law plus neutral reflection component; PL+E: absorbed power law plus absorption edge; Leaky+L: leaky model plus emission line.

to be not acceptably fitted by this model. We did not find these 11 sources to share any common spectral characteristic. For 9 of them, we found that no additional component significantly improved the fit, and we accordingly assume the simple absorbed power-law fit as our best fit and used the data in Table 3 in the subsequent analyses. For the remaining two sources, XBSJ021822.2-050615 and XBSJ153456.1+013033, a leaky absorber plus an emission line and a black body, respectively, did improve the fit significantly, consequently we consider these models as our best-fit model although the probability is still $<10 \%$.

\section{Intrinsic absorption}

We measured intrinsic absorption in excess of the Galactic one for 119 sources, 88 type 1 and 27 type 2 AGN. This absorption is significant (F-test $>95 \%$ ) in 56 cases (30 type 1 and 26 type 2 AGN). For the remaining 65 sources for which the 

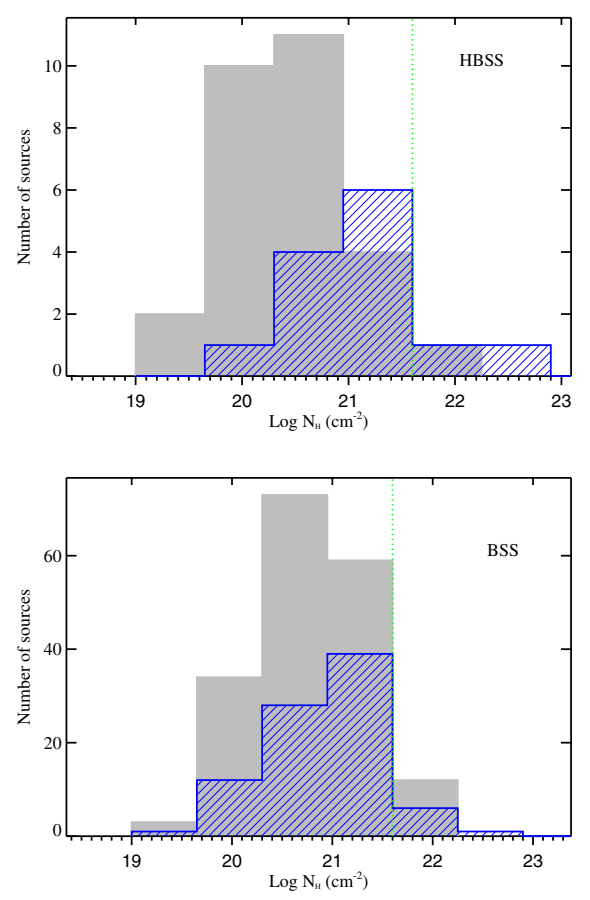
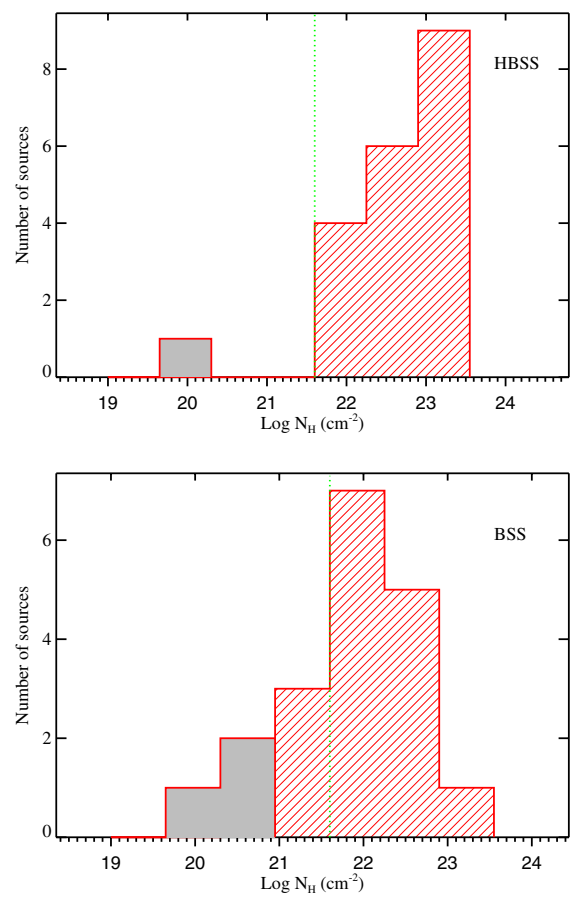

Fig. 2. Intrinsic absorption distribution corresponding to the HBSS (top) and BSS (bottom). Left and right panels correspond to type 1 and type 2 AGN, respectively. Dashed histograms correspond to detected intrinsic absorption, whereas filled histograms correspond to upper limits. Vertical dotted lines mark the threshold used to distinguish between absorbed and unabsorbed sources following the prescription of Caccianiga et al. (2007). significance is below 95\%, the measured amount of absorption is very low, except for one case, XBSJ161820.7+124116. The low significance in this case is likely owing to the extremely low number of counts in the available spectrum. The intrinsic absorption distribution for both studied samples is shown in Fig. 2.

In 17 cases ( 3 type 1 and 14 type 2 AGN) and because of the poor statistics, we fixed the value of $\Gamma$ to 1.9 (which corresponds to the average value found for unabsorbed AGN) to better constrain the intrinsic absorption. For 7 out of these 17 AGN, $\Gamma$ could be determined (although with large errors), but turned out to be flatter than the flattest $\Gamma$ found for unabsorbed AGN $(\Gamma \sim 1.5)$. This is probably owing to the low statistics available for the spectral analysis, which do not allow us to adequately constrain at the same time both the spectral index and the intrinsic absorption in these cases. We note here that the best fit $N_{\mathrm{H}}$ obtained with free $\Gamma$ is usually within the reported errors obtained when fixing $\Gamma=1.9$; furthermore the variation of $N_{\mathrm{H}}$ is such that this problem does not have any effect on the X-ray source classification used here (absorbed vs. unabsorbed) or on the $N_{\mathrm{H}}$ distribution.

\subsection{X-rays versus optical absorption}

Following the criteria described in Caccianiga et al. $(2008)^{4}$, we defined a sourced as absorbed if the measured intrinsic column density is higher than $4 \times 10^{21} \mathrm{~cm}^{-2}$. According to this criterion, we find that 31 sources are absorbed, 8 type 1 (3\% of type $1 \mathrm{AGN})$ and 23 type $2 \mathrm{AGN}(80 \%$ of type $2 \mathrm{AGN})$. We find that the fraction of absorbed sources and the amount of absorption is much higher for type 2 AGN than for type 1 AGN, in agreement with the AGN unified picture. However, there is a number of sources that do not match within this scenario, 8 type 1 AGN are absorbed (3\% total) and 6 type 2 AGN are

\footnotetext{
${ }_{4}$ The optimum dividing line between optical type $1 / 2$ classification is found to correspond to an optical extinction of $A_{V} \sim 2$ mag, which, assuming a Galactic $A_{V} / N_{\mathrm{H}}$ ratio, implies a column density of $N_{\mathrm{H}} \sim 4 \times$ $10^{21} \mathrm{~cm}^{-2}$ in X-rays.
}

unabsorbed (20\% total). Nonetheless, for half of the absorbed type 1 AGN, the errors in the intrinsic column density are consistent with these sources being unabsorbed. It is important to note that the expected intrinsic column density derived from optical reddening depends on the assumed gas-to-dust ratio. If the intrinsic gas-to-dust ratio differs significantly from the Galactic value (the standard gas-to-dust ratio that is usually used), small differences between the expected value from optical observations and the measured value from X-rays are expected. Three of the unabsorbed type 2 AGN are consistent with being absorbed within errors, but only by low amounts of intrinsic absorption $\left(\leq 10^{22} \mathrm{~cm}^{-2}\right)$. This implies that the optical/X-ray type mismatch could be more common for sources that are optically classified as type 2 AGN. However, it is difficult to quantify how frequent the "mismatches" between optical and X-ray classification are.

In an X-ray selected sample, the least biased way to estimate the exceptions to the unified models is to compute the fraction of optically classified type $1 / 2$ AGN among the absorbed/unabsorbed sources. Elusive AGN have to be removed because their classification is derived from X-rays. We also excluded those absorbed/unabsorbed sources that could be unabsorbed/absorbed within errors. In this way, we found that there are only four type 1 AGN among "truly" absorbed sources and two type 2 AGN among "truly" unabsorbed sources. Therefore, the resulting fraction of exceptions to the unified models for the XBS AGN sample turns out to be of $\sim 3 \%$. For the HBSS only, which is almost completely identified, we find that the fraction of type 2 AGN among unabsorbed sources is only $3 \%$. A similarly low value of $1 \%$ is obtained for the BSS. For the fraction of type 1 AGN among absorbed sources, we find 17\% for the HBSS, whereas for the BSS it turns out to be $\sim 30 \%$. However, if we take into account the larger number of unidentified sources on the BSS, seven of which are probably absorbed AGN (see discussion at the end of this section), this number could decrease to match the one obtained for the HBSS.

To compare our findings with previously reported results, we selected a $10^{22} \mathrm{~cm}^{-2}$ limit, which is the one that is usually 
used by other authors, to separate between absorbed and unabsorbed sources. Using this limit and considering the total XBS sample, we computed a fraction of unabsorbed type 2 AGN among the total number of type 2 AGN of $\sim 36 \%$. This number agrees with reported values of unabsorbed type 2 AGN shown in Panessa \& Bassani (2002) and Akylas \& Georgantopoulos (2009) ( 10-30\% and 20\%, respectively). However, it has to be pointed out that none of the reported fractions in Panessa \& Bassani (2002) and Akylas \& Georgantopoulos (2009) has been derived from complete samples. For example, if we consider only the HBSS, which is almost completely identified and that it is less biased against absorbed sources, the value decreases from $36 \%$ to $20 \%$; this is expected to be a more reliable fraction than that computed using the total XBS sample. Moreover, errors in the resulting $N_{\mathrm{H}}$ values are not usually considered either. If we remove unabsorbed type 2 AGN that could be absorbed within $90 \%$ confidence errors, the fraction decreases to $\sim 5 \%$. Therefore, caution must be exercised when computing the fraction of exceptions to unified models; these fractions have probably been overestimated in the past.

The existence of unabsorbed type 2 AGNs has no clear explanation so far. Some recent models (Elitzur \& Shlosman 2006; Nicastro 2000) show that the BLR could not form under particular condition. For instance, it has been proposed that the BLR may disappear below bolometric luminosities of $\sim 10^{42} \mathrm{erg} \mathrm{s}^{-1}$ (Elitzur \& Shlosman 2006) or below a critical accretion rate $\left(L_{\mathrm{bol}} / L_{\mathrm{Edd}} \sim 1-4 \times 10^{-3}\right.$ for SMBH masses ranging from $10^{6}$ to $10^{9}$ solar masses; where $L_{\text {bol }}$ and $L_{\text {Edd }}$ are the bolometric and Eddington luminosities, respectively, Nicastro 2000). Nevertheless, the range of luminosity and accretion rates covered by the unabsorbed type 2 AGN in our sample $\left(L_{\mathrm{X}}\right.$ from $10^{42}$ to $\sim 2 \times 10^{44} \mathrm{erg} \mathrm{s}^{-1}$ and accretion rates from $\sim 10^{-3}$ up to $\sim 1$, Caccianiga et al., in prep.) make these interpretations not applicable to the sources of the XBS sample.

A possible alternative explanation is that unabsorbed type 2 AGN are indeed Compton-tick (CT) i.e. sources where the amount of intrinsic absorption is so high (above $10^{24} \mathrm{~cm}^{-2}$ ) that the absorption cut-off falls outside the observed spectral range. Using X-ray data limited in the $2-10 \mathrm{keV}$ energy band, it would not be possible to compute the actual column density and we would end up with an optically type 2 AGN with no sign of absorption in the X-rays. The unabsorbed type 2 AGN in our sample are XBSJ012057.4-110444, XBSJ031146.1550702, XBSJ100032.5+553626, XBSJ141235.8-030909, XBSJ230522.1+122121 and XBSJ221951.6+120123. XBSJ100032.5+553626 is an elusive AGN, whose Comptonthick nature was studied and discarded as a possible explanation in Caccianiga et al. (2007). To test the Compton-thick hypothesis for the remaining five unabsorbed type 2 AGN, we used the diagnostic diagram by Bassani et al. (1999), which make use of the thickness parameter $(T)$ and the $\mathrm{Fe} \mathrm{K} \alpha$ line $E W$ to separate Compton-thick from Compton-thin sources. The thickness parameter represents the ratio between the $2-10 \mathrm{keV}$ observed flux (corrected for Galactic absorption) and the reddening-corrected flux of the $[\mathrm{OIII}] \lambda 5007 \AA$ emission line. Compton-thick sources usually locate at $T<1$ and large $\mathrm{Fe} \mathrm{K} \alpha$ equivalent widths. The values for the [OIII] fluxes used here were computed following the prescription and assumptions discussed in Caccianiga et al. (2007). Regarding the $\mathrm{Fe} \mathrm{K} \alpha E W \mathrm{~s}$, only upper limits (at the $90 \%$ confidence limit) could be derived; we assumed in all cases an unresolved neutral emission line centered at $6.4 \mathrm{keV}$.

Our results for the five sources in consideration here are plotted in Fig. 3; obviously all our sources are well above the $F_{\mathrm{X}} / F_{\text {[OIII] }}=1$ limit. The computed [OIII] fluxes are not

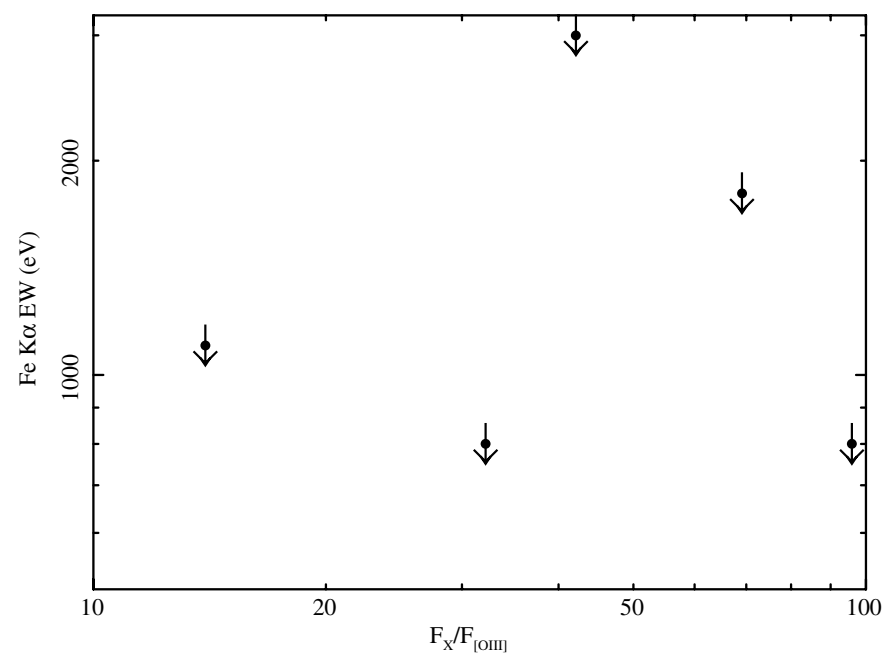

Fig. 3. Fe K $\alpha E W$ versus the thickness parameter $T=F_{\mathrm{X}} / F_{\text {[OIII] }}$ for the 5 unabsorbed type 2 AGN. Vertical arrows correspond to upper limits on the $\mathrm{Fe} \mathrm{K} \alpha E W$.

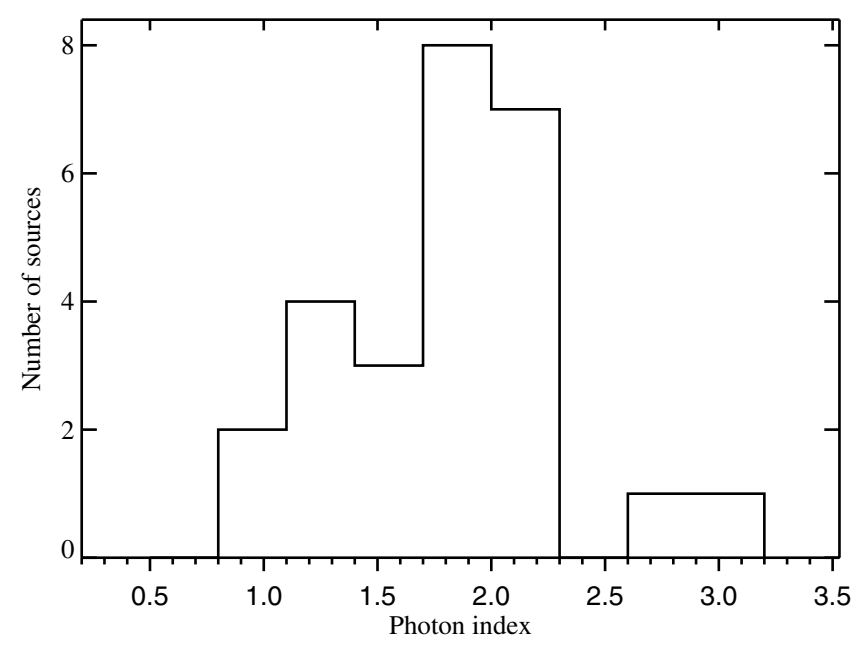

Fig. 4. Photon index distribution for the 27 unidentified sources in the XBS sample.

corrected for extinction from the host-galaxy. Correcting for the host-galaxy extinction would increase the [OIII] fluxes thus decreasing the resulting $T$ values. Nonetheless, the maximum expected extinction $\left(A_{V} \sim 1\right.$ for galaxies with ongoing intense starformation, Calzetti \& Heckman (1999)) is not high enough to place these sources below the $T=1$ limit. We conclude that the CT hypothesis is not a valid explanation for the unabsorbed type 2 AGN in the XBS sample. Further investigations are thus required to understand the nature of these intriguing sources.

Finally the observed fractions of absorbed AGN (number of absorbed AGN to total number of AGN) are $\sim 35 \%$ for the HBSS and $\sim 7 \%$ for the BSS $(34 \%$ and $6 \%$, respectively, if we do not take into account those sources that are consistent with being unabsorbed within errors). This difference is expected because the selection at higher energies is less affected by obscuration, therefore the HBSS is much more efficient in selecting absorbed sources. To investigate how the larger number of unidentified sources in the BSS could affect this, we performed a simple power law X-ray spectral fit over the unidentified AGN in the XBS sample. The resulting photon index distribution is shown in Fig. 4. We can see that seven (including the two unidentified 
A. Corral et al.: The X-ray spectral properties of the AGN population in the XMM-Newton bright serendipitous survey
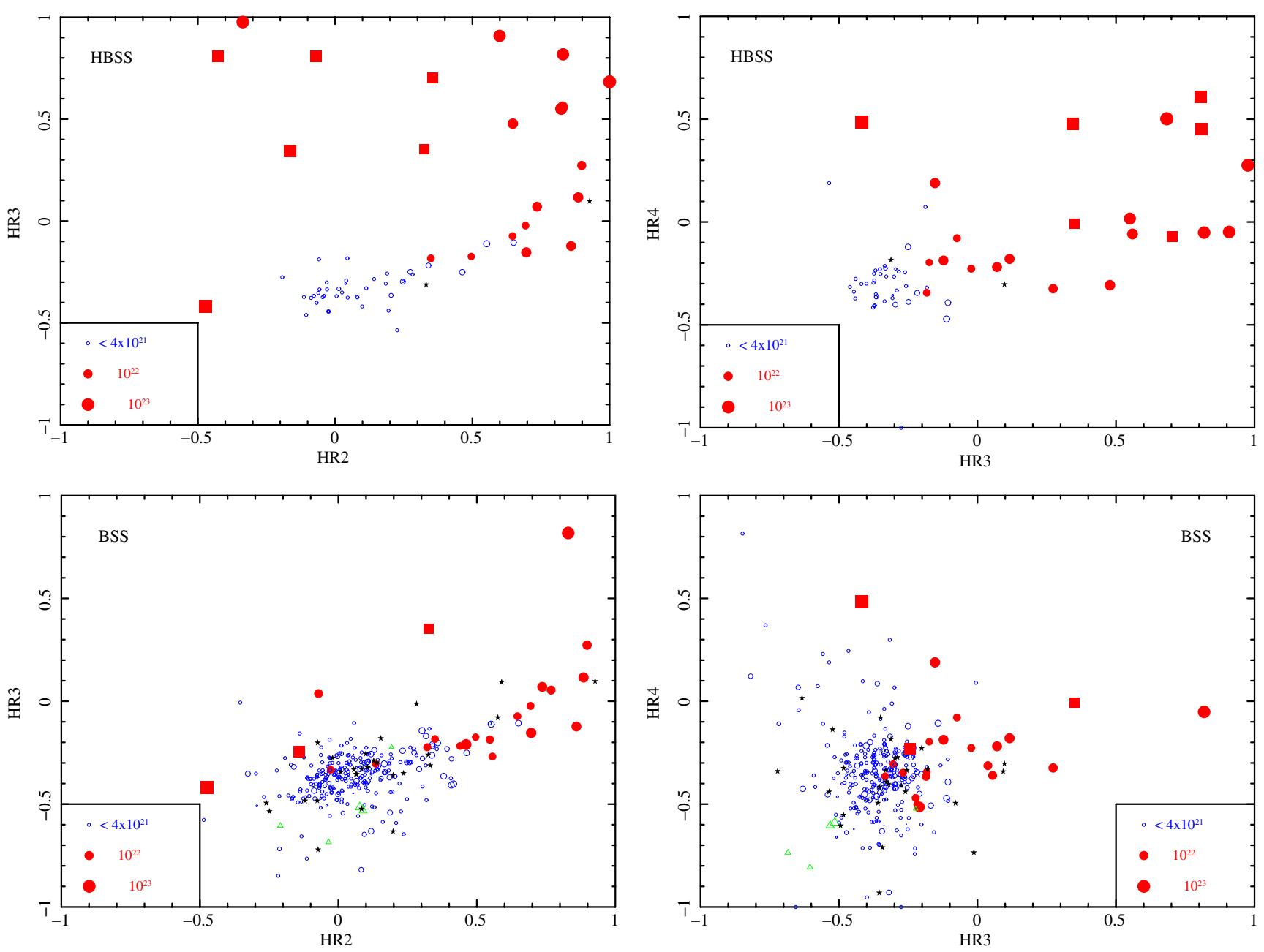

Fig. 5. Hardness ratios for the unabsorbed (open circles) and absorbed (filled circles) identified sources corresponding to the HBSS (top panels) and the BSS (bottom panels), the circle sizes are proportional to the measured intrinsic absorption. Stars correspond to unidentified sources. Filled squares correspond to sources whose best fit is a leaky model.

sources in the HBSS) out of the 27 unidentified sources display a photon index below 1.5. If these seven sources turn out to be absorbed AGN, the fraction of absorbed AGN in the BSS sample would increase to $10 \%$.

\subsection{Hardness ratios}

Another way to estimate the source type by using X-rays is to compute the hardness ratios (X-ray colors). We extracted the hardness ratios HR2, HR3, and HR4 from the 2XMM-Newton catalog 5 (Watson et al. 2009), which are defined as

$\mathrm{HR} n=\frac{\mathrm{CR}_{n+1}-\mathrm{CR}_{n}}{\mathrm{CR}_{n+1}+\mathrm{CR}_{n}}$,

where $\mathrm{CR}_{n}$ is the "vignetting"-corrected count rate in the energy band $n$. The 2-5 energy bands correspond in our case to the count rates in the $0.5-1.0,1.0-2.0,2.0-4.5$, and $4.5-12.0 \mathrm{keV}$ energy bands, respectively. To compare them with the unidentified sources, the hardness ratios for the identified AGN are plotted along with the ones for the unidentified sources in Fig. 5. For clarity errors are not plotted in this figure given the large number of sources. To see how the hardness ratios relate to

\footnotetext{
5 http://xmmssc-www.star.le.ac.uk/Catalogue/2XMM/
}

the fitted absorption, we made the symbol sizes proportional to the measured intrinsic column density. Filled squares refer to sources for which the best-fit model is a leaky model. If we do not take into account these latter sources, we can see how the most absorbed sources concentrate in the upper right in the HR3 vs. HR2 figures. For the HBSS, one of the unidentified sources is clearly within that region, whereas the remaining one is not, although it could be moderately absorbed given its photon index from the power-law fit. For the BSS, only about two lie within that region. Evidently also the intrinsic absorption seems to increase as the hardness ratio HR3 increases.

In Fig. 6 the measured column densities are plotted against HR3. Obviously there seems to be a correlation between the amount of intrinsic absorption and HR3 for absorbed sources, again not taking into account sources with a leaky shape. To derive an estimate of the intrinsic column density for unidentified sources, we fitted a linear model to this correlation. In this way, an intrinsic column density can be estimated even when the X-ray data quality is too poor to carry out a reliable spectral analysis. We performed the fit in two different ways. The first one was to fit a linear model by using $\chi^{2}$ statistics. We considered only those sources with a detected value of the intrinsic column density higher than $4 \times 10^{21} \mathrm{~cm}^{-2}$, i.e. absorbed sources, and we excluded sources with a leaky shape. 


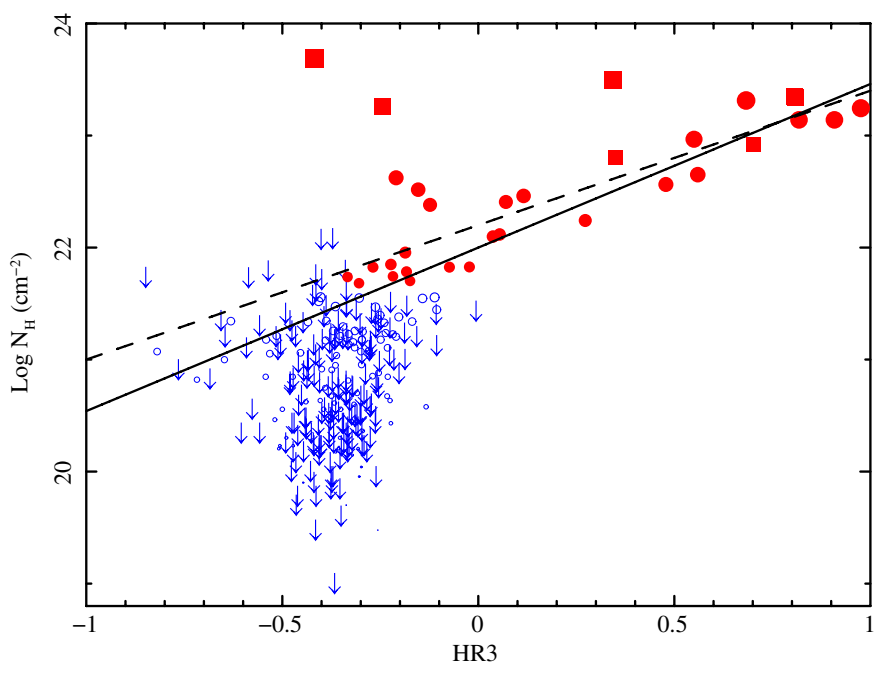

Fig. 6. Intrinsic column density against HR3 for unabsorbed (open circles) and absorbed (filled circles) sources. Filled squares correspond to sources whose best fit is a leaky model. Dashed and solid lines correspond to different fits to the observed correlation (see text for details).

This selection criterion resulted in a total of 25 sources. With a Spearman rank correlation analysis we confirmed a strong correlation $(\rho=0.82$, probability $=0.0001)$. The resulting fitted relation corresponds to the dashed line in Fig. 6 and it is

$\log \left(N_{\mathrm{H}}\right)=22.2( \pm 0.2)+1.2( \pm 0.2) \mathrm{HR} 3$.

Our second approach was to use all sources with a column density or upper limit above the $4 \times 10^{21} \mathrm{~cm}^{-2}$ threshold and also all unabsorbed sources whose intrinsic column densities values were consistent with this limit within $90 \%$ errors, which were 85 sources in total. To perform this analysis, we used the ASURV package (Astronomy Survival Analysis, Isobe \& Feigelson 1990; which implements the methods presented in Isobe \& Feigelson 1986). Applying a Spearman rank analysis, including the upper limits, we found again that there is a strong correlation between the intrinsic column density and HR3 $(\rho=0.84$, probability $<0.0001)$. We performed linear regression with the parametric EM algorithm, solid line in Fig. 6, which assumes Gaussian residuals as in $\chi^{2}$ statistics, obtaining the relation

$\log \left(N_{\mathrm{H}}\right)=22.00( \pm 0.04)+1.46( \pm 0.10) \mathrm{HR} 3$.

Both relations in Eqs. (2) and (3) give similar estimates for the intrinsic column density and can be applied up to redshift $\sim 1$, given the energy bands considered in the computation of HR3, and HR3 $>0$. Making use of these relations, an estimate of the intrinsic column density can be obtained for unidentified sources with small number of collected counts in X-ray surveys. As an example, we found for our unidentified sources that about eight out of the 27 unidentified sources could be absorbed AGN, consistent with what we obtained from the spectral analysis.

\section{Photon index}

Unfortunately, for half of type 2 AGN (14 out of 29) the photon index was fixed to 1.9 during the spectral fit to better constrain the intrinsic absorption, so we restricted our analysis of the power law index to those type $1 \mathrm{AGN}$ in the XBS AGN sample for which we were able to measure $\Gamma, 267$ type 1 AGN. We computed its mean and its intrinsic dispersion making use of Maccacaro et al. (1988) likelihood maximization technique. Because the errors on the photon index are not symmetric, we used the average value for the individual errors in each case. We obtain a mean value of $\langle\Gamma\rangle=2.05 \pm 0.03$ with an intrinsic dispersion of $\sigma=0.26 \pm 0.02$ for the BSS and $\langle\Gamma\rangle=1.98 \pm 0.08$ with an intrinsic dispersion of $\sigma=0.29 \pm 0.05$ for the HBSS, in agreement with previous works (Mateos et al. 2010; Young et al. 2009; Dadina 2008; Mainieri et al. 2007; Page et al. 2006; Mateos et al. 2005a,b). Errors were extracted from the $1 \sigma$ confidence contours. The measured power-law index distribution for the BSS and the HBSS is shown in Fig. 7 along with the computed mean and intrinsic dispersion and their confidence contours. There are three type 1 AGN for which the resulting photon index is $>3$. Two of them are NLSy1s (narrow line Seyfert 1 galaxies), known to show these high values for the photon index, and the remaining one is a Seyfert galaxy with a low number of counts in its X-ray spectra.

To check for possible dependence of the photon index on redshift or luminosity, the values for the individual photon index measurements are presented along with the source redshift and luminosity in Fig. 9. We find an anti correlation between the power-law index and the X-ray luminosity $(\rho=-0.21$, probability $\left.=8 \times 10^{-4}\right)$ and marginally redshift $(\rho=-0.10$, probability $=0.09)$ with the Spearman rank correlation analysis. We also find a correlation with the $0.5-2 \mathrm{keV}$ flux $(\rho=0.15$, probability $=0.01$, which could be caused by undetected intrinsic absorption as pointed out in Mateos et al. (2010). In that work, the authors found a stronger correlation between the photon index and the source redshift, but in their case it mainly started above redshift 2 and our sample only contains 3 sources above that redshift. In our case, we find that the anticorrelation between photon index and luminosity seems to be the strongest one. Moreover, and given that this is a flux-limited sample, the dependence on redshift could be merely caused by the dependence on luminosity. To test this scenario, we selected two narrow luminosity ranges, $10^{43}$ to $10^{44} \mathrm{erg} \mathrm{s}^{-1}$, and $10^{44}$ to $10^{45} \mathrm{erg} \mathrm{s}^{-1}$ (below $10^{43} \mathrm{erg} \mathrm{s}^{-1}$ there are not enough sources to perform a reliable analysis), and applied the same correlation analysis as for the whole type 1 AGN sample. For the lowest luminosity range, which reaches only $z \sim 0.8$, we find that the anti-correlations turns into a correlation between the photon index and redshift $(\rho=0.29$, probability $=0.006)$, while for the high-luminosity bin, which reaches $z \sim 1.5$, we find that the correlation disappears $(\rho=0.06$, probability $=0.69)$. This may imply that the observed correlation between the source photon indices and redshifts is mainly driven by an actual correlation between the photon index and the intrinsic luminosity.

To better explore these correlations, we constructed redshift and luminosity bins by dividing the sample into six bins with an equal number of sources ( 45 sources per bin, 42 in the last bin) and applied the likelihood-maximization technique to each bin. The results are presented in Fig. 9. An anticorrelation between the photon index and redshift and luminosity seems to be present, but it is within the intrinsic dispersion at each redshift or luminosity bin. A similar result is also found in Mateos et al. (2010). These authors pointed out that the hardening of the spectra at higher luminosities and redshifts can be caused by a decrement in the detection efficiency for softer sources and an increment for harder sources, given that the sample is flux-limited.

To further constrain the correlation of the photon index with the intrinsic luminosity, which seems to be the strongest correlation in our case, we divided the sample into two subsamples, the criterion of which was if the intrinsic luminosity was higher or lower than $10^{44} \mathrm{erg} \mathrm{s}^{-1}$. The results are displayed in Fig. 8. 
A. Corral et al.: The X-ray spectral properties of the AGN population in the XMM-Newton bright serendipitous survey
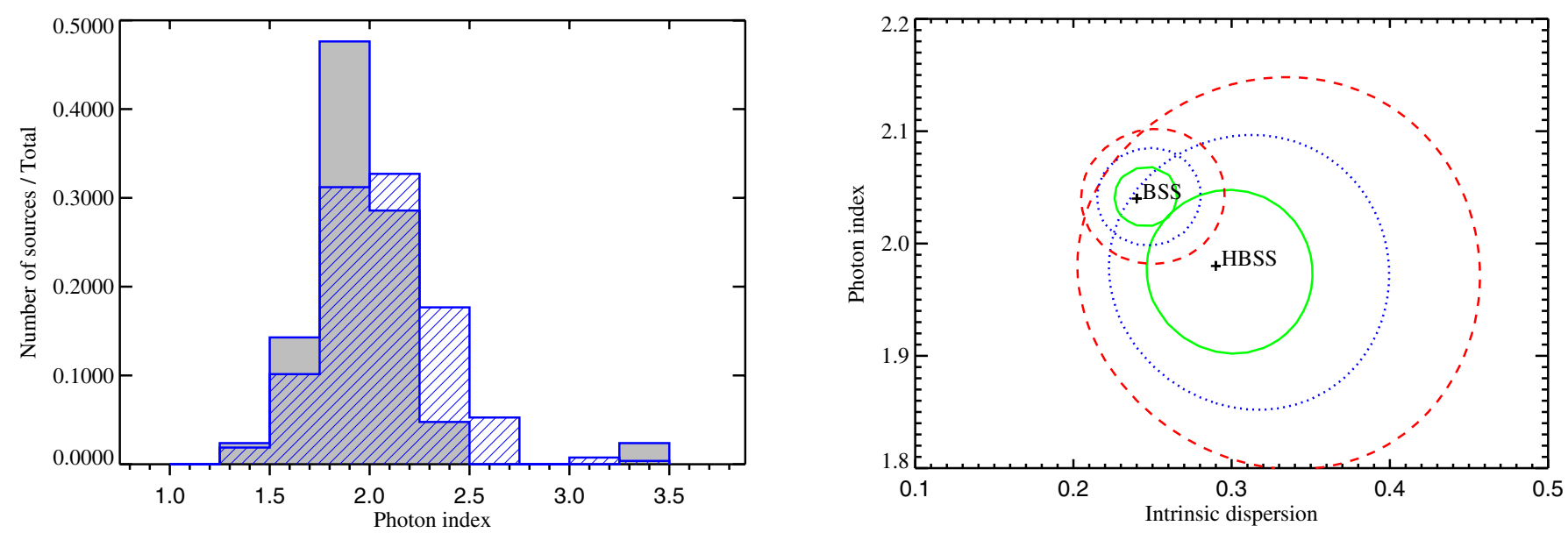

Fig. 7. Left panel: photon index distribution for the BSS (dashed histogram) and the HBSS (filled histogram). Right panel: computed mean photon index and intrinsic dispersion for the BSS and the HBSS along with the $1 \sigma$ (solid line), $2 \sigma$ (dotted line) and $3 \sigma$ (dashed line) confidence contours.
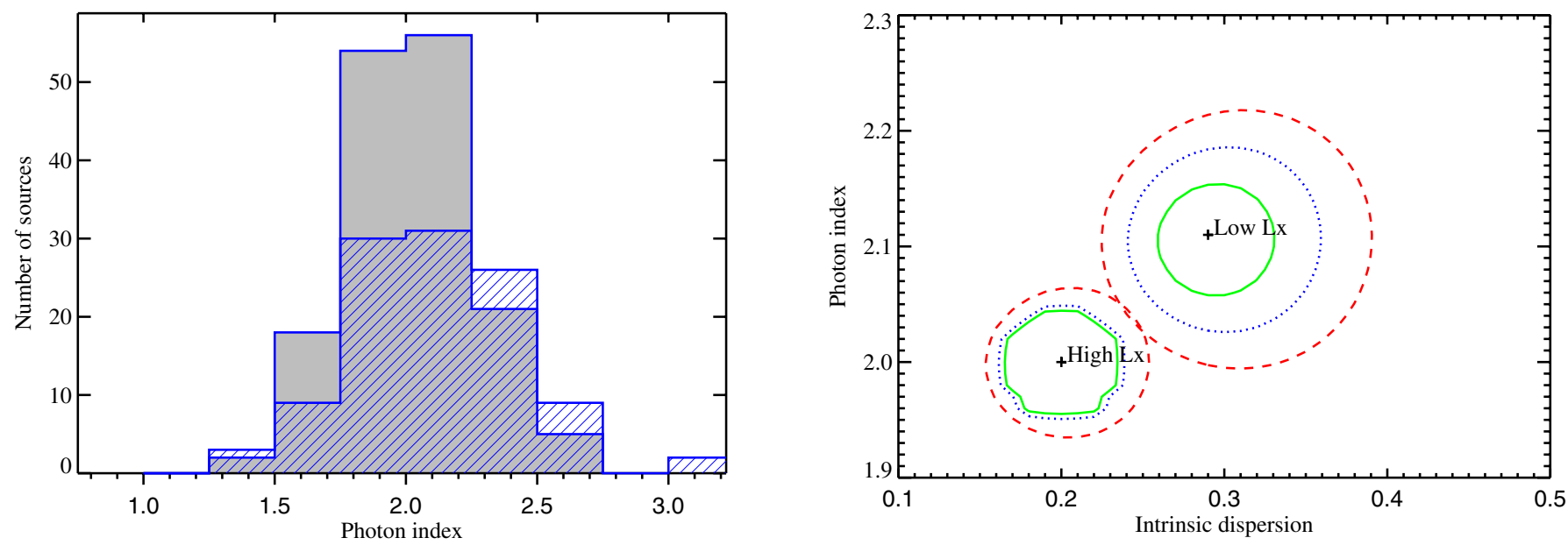

Fig. 8. Left panel: photon index distribution corresponding to the low-luminosity (dashed histogram) and the high-luminosity (filled histogram) subsamples. Right panel: computed mean and intrinsic dispersion for the low- and high-luminosity subsamples along with the $1 \sigma$ (solid line), $2 \sigma$ (dotted line) and $3 \sigma$ (dashed line) confidence contours.

We compute a value of $\langle\Gamma\rangle=2.11 \pm 0.04$ with an intrinsic dispersion of $\sigma=0.29 \pm 0.04$ for the low-luminosity subsample and $\langle\Gamma\rangle=2.00 \pm 0.05$ with an intrinsic dispersion of $\sigma=0.20 \pm 0.04$ for the high-luminosity subsample. The mean and intrinsic dispersion results are different almost at the $3 \sigma$ confidence level, although the main difference seems to be on the intrinsic dispersion. Applying a Kolmogorov-Smirnov (K-S) test, we find that the probability for both distributions to be drawn from the same parent distribution is only $\sim 1 \%$. The opposite is found in Bianchi et al. (2009a), who analyzed high-quality X-ray spectral data and found that Seyfert galaxies show a flatter photon index than quasars.

In principle, the observed correlation of $\Gamma$ with the luminosity could be owing to the presence of an undetected reflectionemission component that becomes increasingly important with the luminosity. In Sect. 7 we show that this correlation is present also when we analyze the average spectra, where the reflection component is already accounted for. This result excludes that the flattening of $\Gamma$ with the luminosity is due to the reflection component.

Because the type 1 AGN sample we used in this particular analysis could be contaminated by radio-loud (RL) sources, which are expected to have a flatter photon index on average (Reeves et al. 1997; Reeves \& Turner 2000), we performed a safety test. Making use of the NVSS/XBS cross-correlation and analysis presented in Galbiati et al. (2005), we removed all RL sources within these 267 type 1 AGN, which were 14 sources, and applied the same likelihood analysis by dividing into high- and low-luminosity AGN. We obtained the same result as for the whole sample, the only difference was a small decrement on the intrinsic dispersion for the high-luminosity subsample $(\sigma=0.19 \pm 0.04)$, and the two samples were still different almost at the $3 \sigma$ level.

\section{Soft-excess emission}

We say a source shows a soft-excess emission when the extrapolated 2-10 keV power-law fit displays systematic positive residuals at low energies. We find that 35 AGN out of the 41 sources that were not well-fitted by an absorbed power law display a softexcess. For 29 out of these 35 AGN, we are able to find an additional component that significantly improves the simple powerlaw fit, as measured by F-test. Assuming a fraction of spurious detections of 0.05 , given our F-test significance limit of $95 \%$, 

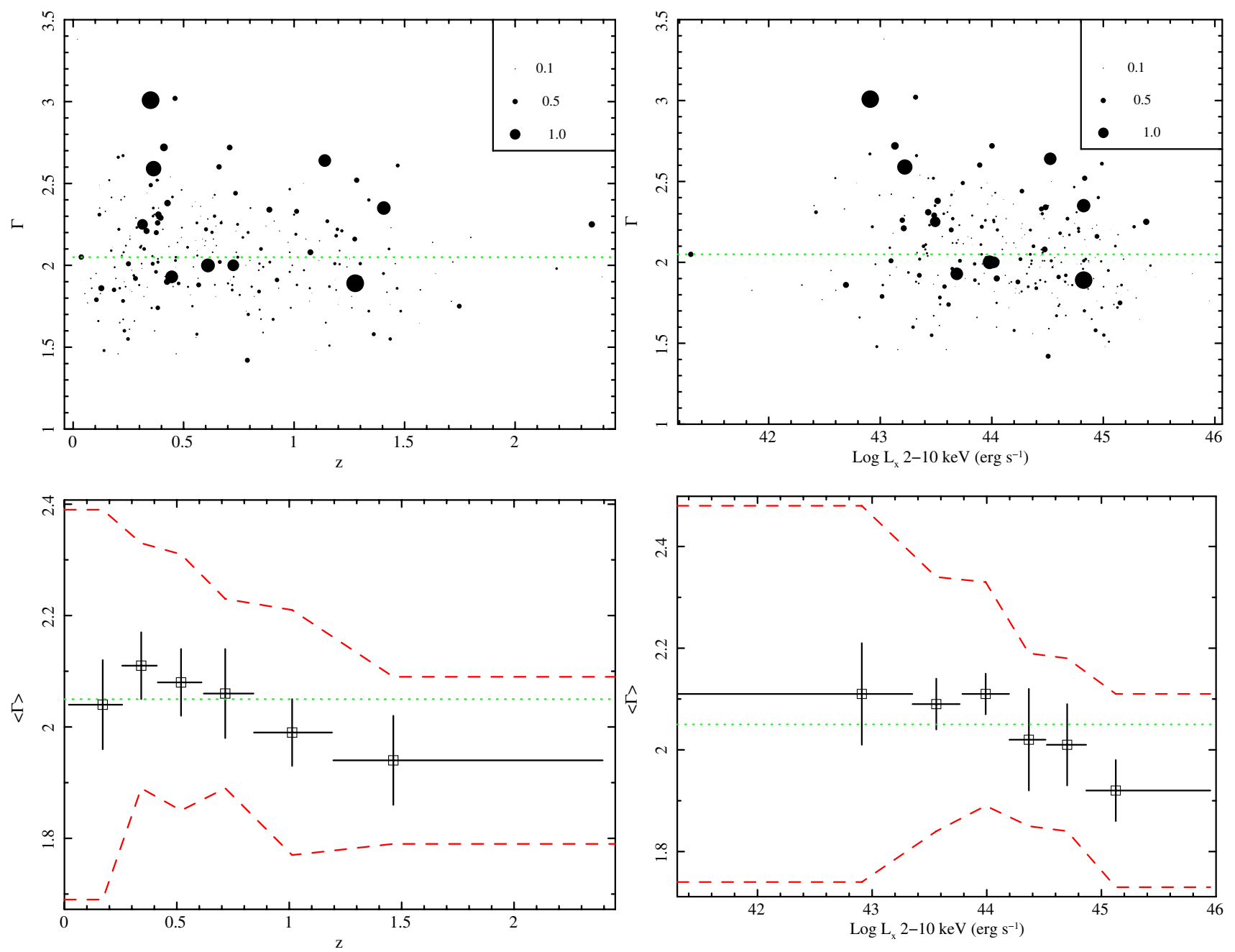

Fig. 9. Top panel: type $1 \mathrm{AGN}$ photon index versus redshift (left) and luminosity (right). The size of the circles indicates the size of the errors on the photon index. The horizontal dotted line corresponds to the average photon index for the whole sample. Bottom panel: type 1 AGN average photon index versus redshift (left) and luminosity (right) for the luminosity and redshift bins. The error bars correspond to the mean error at $1 \sigma$ confidence level, whereas the dashed lines mark the values for the intrinsic dispersion at each bin.

this number corresponds to $5_{-4}^{+2} \%$ of the total XBS AGN sample $\left(14_{-7}^{+9} \%\right.$ for the HBSS and $4_{-2}^{+2} \%$ for the BSS). If we only take into account sources below $z=0.5$ (beyond that value most of the soft-excess emission is redshifted outside the EPIC energy range) the fraction of sources increases to $11_{-5}^{+6} \%$ for the XBS sample $\left(20_{-10}^{+12} \%\right.$ for the HBSS and $9_{-4}^{+6} \%$ for the BSS), a value closer to the reported values in recent works (Mateos et al. 2010; Bianchi et al. 2009a), although still lower. It should be noted that our computed value has to be considered as a lower limit because we did not search for soft-excess emission for all our sources, but only for the ones for which a simple power law gives a probability $<10 \%$. This could also explain why the fraction of sources showing soft-excess is larger for the HBSS than for the BSS. The difference in the fraction of detected soft-excess in both samples is likely caused by differences in the data quality. The collected number of counts for the HBSS spectra is larger on average than for the BSS. As the spectral quality increases, it becomes easier to detect and characterize additional components. Undetected soft-excess would also increase the value for the measured photon index, and this in turn could be contributing to increase the computed average photon index for the BSS and resulting in a higher value than the one for the HBSS.
In the case of absorbed AGN and thanks to Chandra and $X M M-N e w t o n$ grating spectra, this soft-excess is known to be associated to scattered emission hundreds of pc far form the central source, likely by the NLR clouds (see for example Bianchi et al. 2006). Indeed, all absorbed AGN that display soft-excess, five type 2 and two type 1 AGN, are best-fitted either by a leaky model or by a power law plus a thermal component that could arise from the host galaxy given its low luminosity.

The case of unabsorbed AGN is more complex. Soft-excess emission has usually been attributed to the hard tail of the thermal emission from the accretion disk or to optically-thick comptonization of EUV disk photons (Ross et al. 1992; Shimura \& Takahara 1993), but these models are unable to explain either the higher temperatures usually detected or the fact that these temperatures seem not to vary with AGN properties such as the intrinsic luminosity. A recent model, also invoking continuum emission, explains this soft-excess emission via optically-thin comptonization of the disk photons (Kawaguchi et al. 2001), which would explain the non-dependence on the source luminosity. Two alternative models, based on atomic processes within the accretion disk, have been proposed in recent works: the soft-excess emission could come form relativistically blurred 
Table 5. Average spectra fit results.

\begin{tabular}{lccccccc}
\hline \hline Sample & $\begin{array}{c}N_{\mathrm{H}} \\
10^{22}\end{array}$ & $\Gamma$ & $R$ & $E$ & $\sigma$ & $E W$ & $\chi^{2} /$ d.o.f. \\
& $\left(\mathrm{cm}^{-2}\right)$ & & & & & & \\
& $(2)$ & $(3)$ & $(4)$ & $(5)$ & $(6)$ & $(7)$ & $(8)$ \\
\hline BSS type 1 AGN & $\ldots$ & $2.02_{-0.03}^{+0.04}$ & $0.6_{-0.2}^{+0.2}$ & $6.40_{-0.06}^{+0.04}$ & $<160$ & $110_{-40}^{+30}$ & $21 / 16$ \\
HBSS type 1 AGN & $\ldots$ & $2.00_{-0.07}^{+0.05}$ & $0.9_{-0.2}^{+0.2}$ & $6.44_{-0.04}^{+0.05}$ & $<140$ & $80_{-40}^{+60}$ & $15 / 16$ \\
\hline BSS type 2 AGN & $<1.1$ & $1.9^{f}$ & $1.0_{-0.8}^{+0.7}$ & $6.53_{-0.06}^{+0.14}$ & $<200$ & $200_{-150}^{+150}$ & $13 / 16$ \\
HBSS type 2 AGN & $2.0_{-0.4}^{+0.5}$ & $1.9^{f}$ & $1.0_{-0.3}^{+0.3}$ & $6.42_{-0.13}^{+0.09}$ & $<250$ & $90_{-60}^{+50}$ & $6 / 16$ \\
\hline Unabsorbed AGN & $\ldots$ & $2.10_{-0.03}^{+0.03}$ & $0.5_{-0.1}^{+0.1}$ & $6.40_{-0.06}^{+0.04}$ & $<160$ & $100_{-40}^{+30}$ & $18 / 16$ \\
Absorbed AGN & $1.2_{-0.3}^{+0.4}$ & $1.9^{f}$ & $1.5_{-0.3}^{+0.2}$ & $6.47_{-0.07}^{+0.06}$ & $<160$ & $100_{-50}^{+70}$ & $9 / 16$ \\
\hline Low-luminosity type 1 AGN & $\ldots$ & $2.11_{-0.20}^{+0.10}$ & $0.8_{-0.5}^{+0.8}$ & $6.43_{-0.13}^{+0.06}$ & $<180$ & $110_{-30}^{+30}$ & $12 / 16$ \\
High-luminosity type 1 AGN & $\ldots$ & $2.00_{-0.03}^{+0.03}$ & $0.3_{-0.1}^{+0.1}$ & $6.39_{-0.05}^{+0.04}$ & $<130$ & $80_{-30}^{+30}$ & $25 / 16$ \\
\hline
\end{tabular}

Notes. Columns: (1) sample used to construct the average spectrum; (2) intrinsic column density; (3) photon index; (4) reflection scaling factor; (5) Fe K $\alpha$ central energy; (6) Fe K $\alpha$ width upper limit; (7) Fe K $\alpha$ equivalent width; (8) $\chi^{2}$ to number of degrees of freedom.

(f) Fixed parameter.

reflection from a partially ionized accretion disk (Crummy et al. 2006) or from velocity-smeared absorption from partially ionized material coming from a disk wind (Middleton et al. 2007), although they are indistinguishable at the EPIC energies. Besides, the quality of our data prevents us from applying them in our spectral analysis. Using the additional components described in Sect. 3, we find a great variety of best-fit models within the unabsorbed AGN that show soft-excess in our sample, 27 sources in total: 3 reflection components, 3 ionized absorbers, 13 black body models and two power laws plus an absorption edge. For six of them no acceptable fit was found, therefore the simple power law model was adopted as the best-fit model.

As mentioned before, when the soft-excess in unabsorbed AGN is modeled with a black body model, a value of $k T \sim$ $0.1 \mathrm{keV}$ is obtained that does not depend on the source flux, redshift or luminosity. To compare our results with previous works, we also attempted to fit a black body plus a power law to all unabsorbed sources with a soft-excess (see Table 6). This improved the simple power-law fit, F-test $>95 \%$, in all but 6 cases, 21 sources in total. The values obtained for the black body temperature are presented in Fig. 10 against the $2-10 \mathrm{keV}$ luminosity. By using a Spearman rank correlation analysis, we found a significant correlation between the black body temperature and the source's luminosity $(\rho=0.60$, probability $=0.004)$. This can be because higher luminosity sources are at higher redshifts, given that the sample is flux-limited, which means that the softemission is shifted outside the observed energy range. Therefore, at higher luminosities only black body components of higher temperatures can be detected. Indeed, if we remove sources at $z>0.5$, the correlation disappears $(\rho=0.06$, probability $=0.78)$. Therefore, and given the present statistics, we cannot confirm if there is an actual correlation between the black body temperature and the hard X-ray luminosity, although our results suggest that high black body temperatures can only be reached by sources with high intrinsic luminosities.

\section{Average spectrum}

A narrow $\mathrm{Fe} \mathrm{K} \alpha$ emission line (6.4-6.9 keV, depending on ionization state of the material) is almost ubiquitously observed in good quality X-ray spectra of AGN (Nandra et al. 2007;

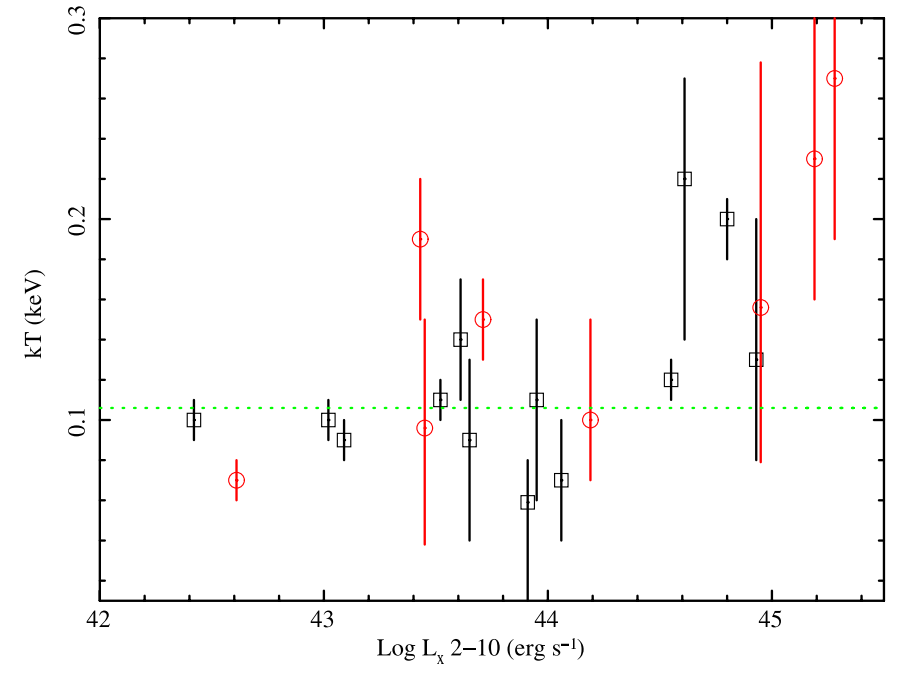

Fig. 10. Black body temperature versus $2-10 \mathrm{keV}$ luminosity for unabsorbed AGN. Squares correspond to the temperature values if a power law plus a black body component is considered the best-fit model, whereas circles correspond to the temperature values in the remaining unabsorbed AGN with a soft-excess emission. The horizontal dotted line corresponds to the weighted mean.

Bianchi et al. 2009b). To measure a broad and/or a relativistically broadened component requires an even better quality (Guainazzi et al. 2006). We searched for this emission line in our sample, but its presence is only suggested in $\sim 20$ sources and its parameters are only well constrained in a couple of cases, all corresponding to a narrow $\mathrm{Fe} \mathrm{K} \alpha$ line. To improve the SNR and to detect spectral features that would remain hidden otherwise, such as the $\mathrm{Fe} \mathrm{K} \alpha$ line, we averaged the whole sample following the process described in Corral et al. (2008). The basic steps of this averaging method are

- Selecting those individual spectra, pn or MOS, with more than 80 counts in the total $0.2-12 \mathrm{keV}$ EPIC band. For the XBS AGN sample, this means to exclude 12 sources, 8 type 1 and 4 type 2 AGN. 


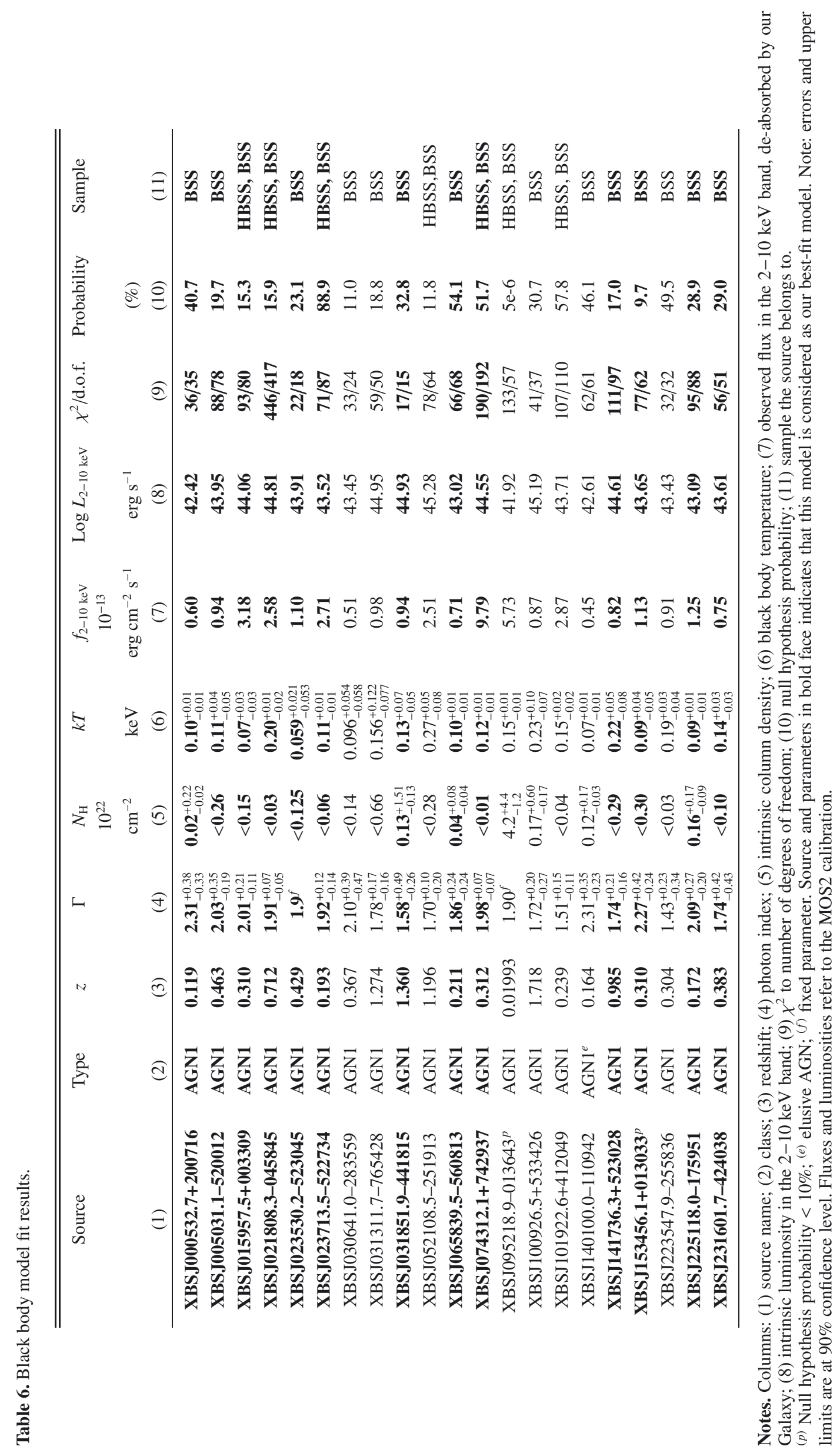


A. Corral et al.: The X-ray spectral properties of the AGN population in the XMM-Newton bright serendipitous survey

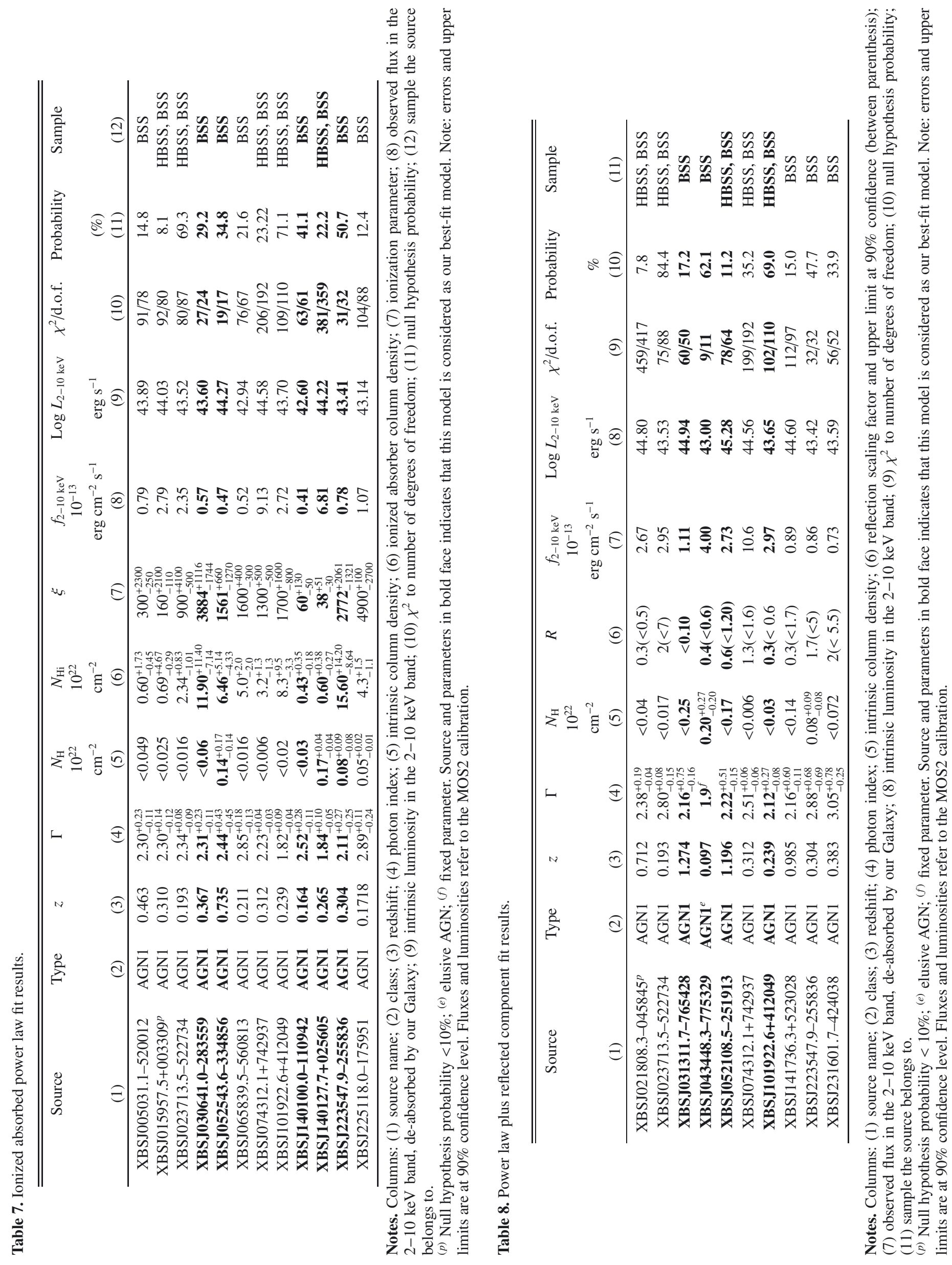



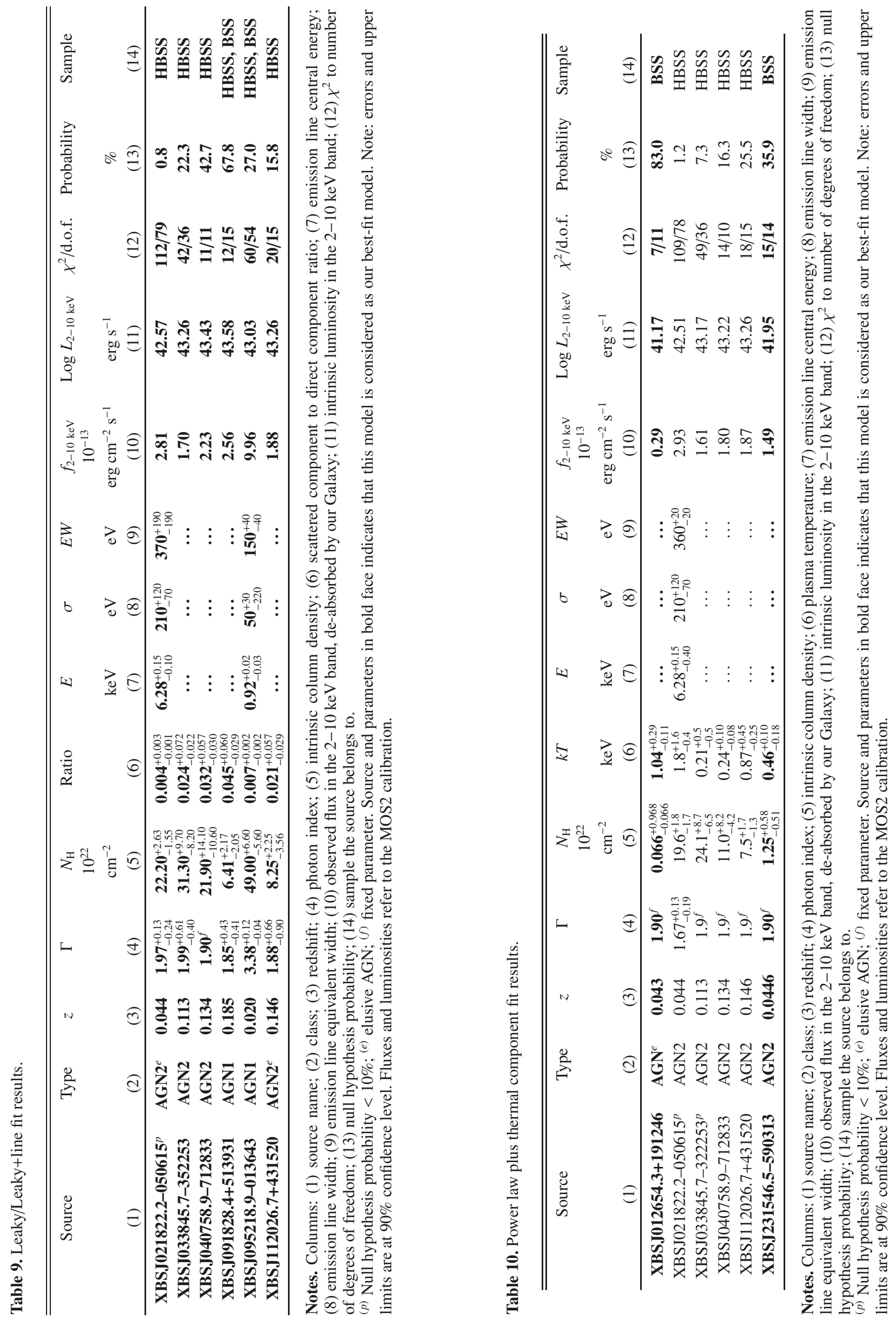
- Fitting pn and MOS spectra for each source individually by using a simple absorbed power-law model and leaving the power-law index, intrinsic column density, and the normalization as free parameters.

- Obtaining the incident spectra, i.e. before entering the detectors, in flux units $\left(\mathrm{keV} \mathrm{cm}^{-2} \mathrm{~s}^{-1} \mathrm{keV}^{-1}\right)$ by using the parameters from the previous spectral fit.

- Correcting for the absorption from our Galaxy and shifting to rest frame.

- Rescaling the individual spectra so that every spectrum has the same $2-5 \mathrm{keV}$ rest frame flux.

- Binning every spectrum to a common energy grid so that the final averaged spectrum has at least 1000 counts per bin.

- Averaging by using a standard mean.

As a final step and to quantify the significance of any spectral feature, we used simulations: we simulated each source 100 times by using the fitted model and keeping the same spectral quality as for the real data. By averaging all the simulations we obtained a simulated "continuum" that should account for the average of absorbed power laws. Taking one simulation for each real spectrum and averaging these, we constructed 100 simulated continua from which we can compute $1 \sigma$ and $2 \sigma$ limits by removing the 32 and 5 extreme values at each bin. In this way, we can say that any excursion over or below these limits is detected at $1 \sigma$ or $2 \sigma$ confidence level. By using the simulated "continuum" and the confidence limit and comparing them to our data, we can determine if there are any significant deviations from a power-law shape and estimate their significance. Only the energies in the 2 to $15 \mathrm{keV}$ rest-frame energy band are used in this spectral analysis. For energies below $2 \mathrm{keV}$, the averaging method is highly dependent on the model used to unfold the spectra, and for energies above $15 \mathrm{keV}$ noise becomes too important. The resulting best-fit models and parameters for each case are shown in Table 5.

First, and to compare the two samples under study here, we constructed the average spectra and confidence limits for the type 1 and type 2 AGN within the BSS and HBSS separately. The resulting averaged spectrum, simulated continuum, and confidence limits for the BSS and HBSS samples are shown in Fig. 11.

$B S S$ : for the type $1 \mathrm{AGN}$ we find that the best-fit model consists of a power law of $\Gamma=2.02_{-0.03}^{+0.04}$ plus a narrow Fe $\mathrm{K} \alpha$ line centered at $E=6.40_{-0.06}^{+0.04} \mathrm{keV}$ and equivalent width $E W=$ $110_{-40}^{+30} \mathrm{eV}$ and a reflection component with a reflection factor $R \sim 0.6$. The inclination angle of the reflection component is always fixed to its default value, $i \sim 60 \mathrm{deg}$, because it cannot be determined at the same time as the reflection factor. For the type 2 AGN the best-fit model turns out to be an absorbed power law ( $\Gamma$ fixed to 1.9 ) with an intrinsic column density $N_{\mathrm{H}}<$ $1.1 \times 10^{22} \mathrm{~cm}^{-2}$ plus a narrow emission line $\left(E=6.53_{-0.04}^{+0.14} \mathrm{keV}\right.$, $\left.E W=200_{-150}^{+150} \mathrm{eV}\right)$ and a reflection component $(R \sim 1)$. The fitted intrinsic column density does not represent the actual average absorption of the sources, but it is related to the fraction of absorbed sources among the type 2 AGN as well as their column densities, and as such does not have a meaningful physical interpretation. In any case, and given that not only the statistics are much lower for type 2 AGN but also that we are fitting above $2 \mathrm{keV}$, the column density cannot be very well constrained.

HBSS: for the type $1 \mathrm{AGN}$ we find that the best-fit model consists of a power law of $\Gamma=2.00_{-0.07}^{+0.05}$ plus a narrow Fe $\mathrm{K} \alpha$ line centered at $E=6.44_{-0.04}^{+0.05} \mathrm{keV}$ and equivalent width 
A\&A 530, A42 (2011)
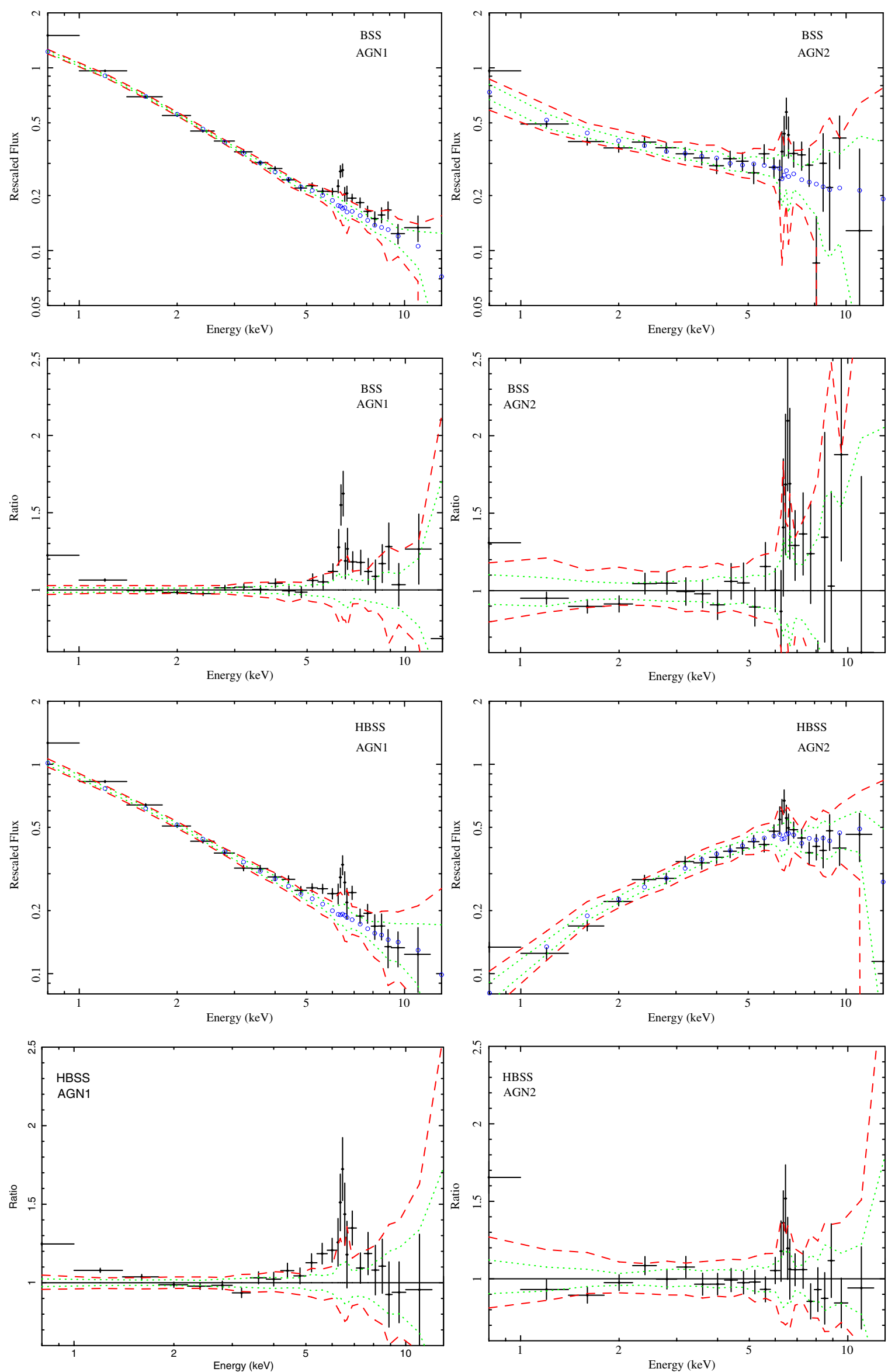

Fig. 11. Type 1 (left) and type 2 (right) AGN average spectrum and average spectrum to simulated continuum ratio (bottom) corresponding to the HBSS and BSS samples. Error bars: real average spectrum, circles: average continuum, dashed line: $2 \sigma$ limit, dotted line: $1 \sigma$ limit. 
A. Corral et al.: The X-ray spectral properties of the AGN population in the XMM-Newton bright serendipitous survey
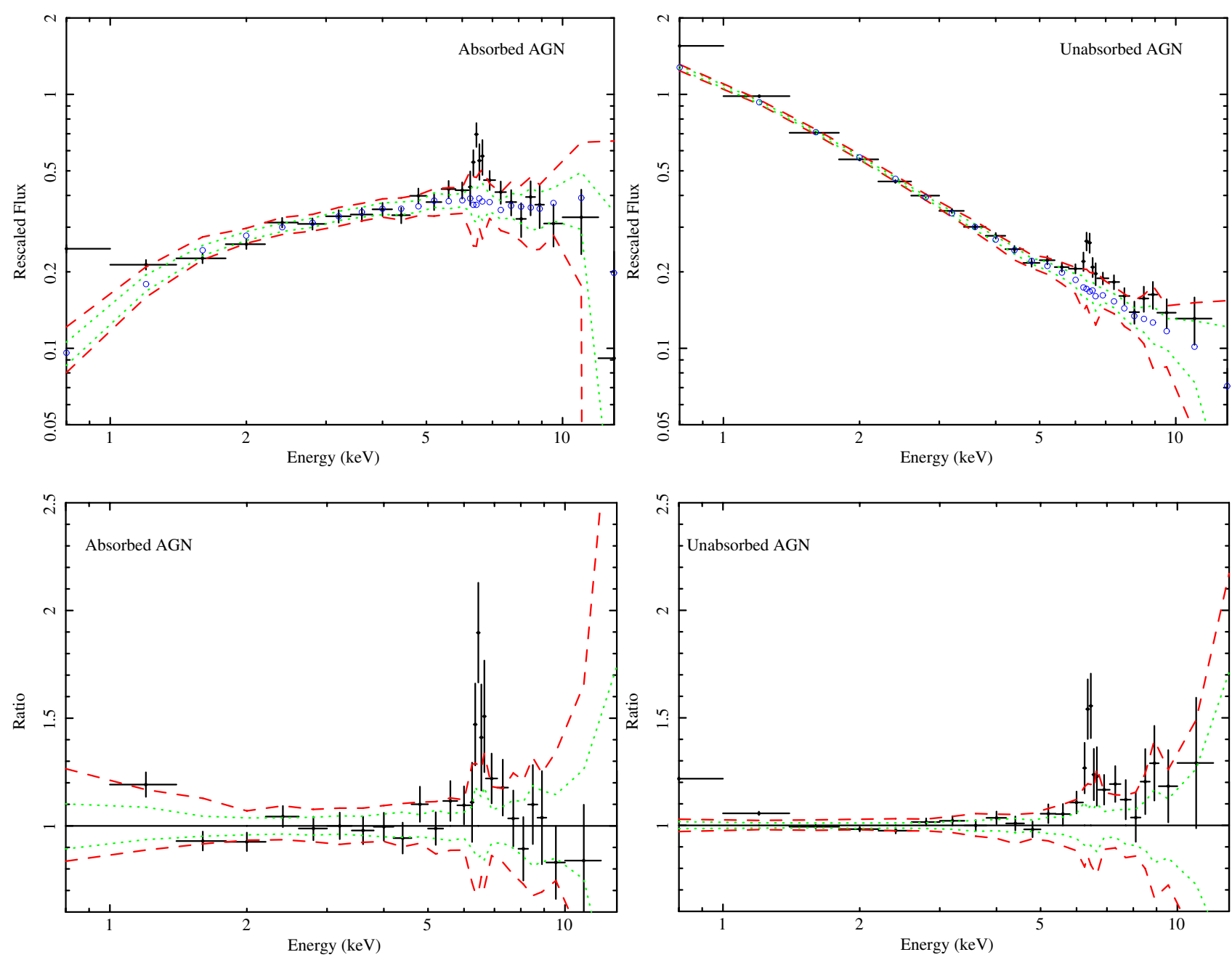

Fig. 12. Average spectrum (top) and average spectrum to simulated continuum ratio (bottom) corresponding to the absorbed (left) and unabsorbed (right) sources. Error bars: real average spectrum, circles: average continuum, dashed line: $2 \sigma$ limit, dotted line: $1 \sigma$ limit.

$E W=80_{-40}^{+60} \mathrm{eV}$ and a reflection component with a reflection factor $R \sim 0.9$. For the type 2 AGN the best-fit model turns out to be an absorbed power law ( $\Gamma$ fixed to 1.9 ) with an intrinsic column density $N_{\mathrm{H}} \sim 2 \times 10^{22} \mathrm{~cm}^{-2}$ plus a narrow emission line $\left(E=6.42_{-0.13}^{+0.09} \mathrm{keV}, E W=90_{-60}^{+50} \mathrm{eV}\right)$ and a reflection component $(R \sim 1)$.

The small differences between type 2 AGN average spectra for the BSS and HBSS are caused by the larger number of absorbed sources for the HBSS. This is expected because selecting at harder energies makes the sample less biased against absorbed sources. The relatively low value for the average column density for the type 2 AGN in the BSS is consistent with it being due to contribution of unabsorbed type $2 \mathrm{AGN}$, which are more numerous in the BSS (which contains six unabsorbed type 2 AGN out of 19 type 2 AGN) than in the HBSS (which only contains one unabsorbed type 2 AGN out of 20). For both samples and AGN classes, the detected $\mathrm{Fe} \mathrm{K} \alpha$ line turns out to be narrow and likely comes from neutral material, i.e., far from the central source.

To better characterize the differences in the spectral shape from absorbed to unabsorbed sources, we divided the whole sample into absorbed (31 AGN) and unabsorbed (274 AGN) sources and constructed the average spectra. The results are shown in Fig. 12.
For the unabsorbed sources we find that the best-fit model consists on a power law of $\Gamma=2.10_{-0.03}^{+0.03}$ plus a narrow Fe K $\alpha$ line centered at $E=6.40_{0.06}^{+0.04} \mathrm{keV}$ and equivalent width $E W=$ $100_{-40}^{+30} \mathrm{eV}$ and a reflection component with a reflection factor $R \sim 0.5$. A relativistic $\mathrm{Fe} \mathrm{K} \alpha$ line is not clearly present and when we attempted to fit one, we did not obtain a significant improvement, and the line parameters resulted in unphysical values (such as a very large inclination angle, $i \sim 80 \mathrm{deg}$, or a extremely high line energy $\sim 8 \mathrm{keV}$ ). From the best-fit model, we estimate an upper limit for the $E W$ of a relativistic $\mathrm{Fe} \mathrm{K} \alpha$ line contribution of $230 \mathrm{eV}$ at the $3 \sigma$ confidence level for a relativistic emission line (Laor 1991) centered at $6.4 \mathrm{keV}$ and inclination angle of $30 \mathrm{deg}$. For the absorbed sources the best-fit model turns out to be an absorbed power law ( $\Gamma$ fixed to 1.9 ) with an intrinsic column density $N_{\mathrm{H}} \sim 1 \times 10^{22} \mathrm{~cm}^{-2}$ plus a narrow emission line $\left(E=6.47_{-0.07}^{+0.06} \mathrm{keV}, E W=100_{-50}^{+70} \mathrm{eV}\right)$ and a reflection component $(R \sim 1.5)$. The main difference between absorbed and unabsorbed sources seems to be a larger amount of reflection in the case of absorbed AGN besides the amount of absorption. This difference is not due to a hidden dependence of the reflection with luminosity because absorbed and unabsorbed AGN in the XBS sample display a very similar luminosity distribution with an average luminosity of $\left\langle L_{X}(2-10 \mathrm{keV})\right\rangle \sim 4 \times 10^{44}$ in both cases and about the same dispersion. The averaging process 

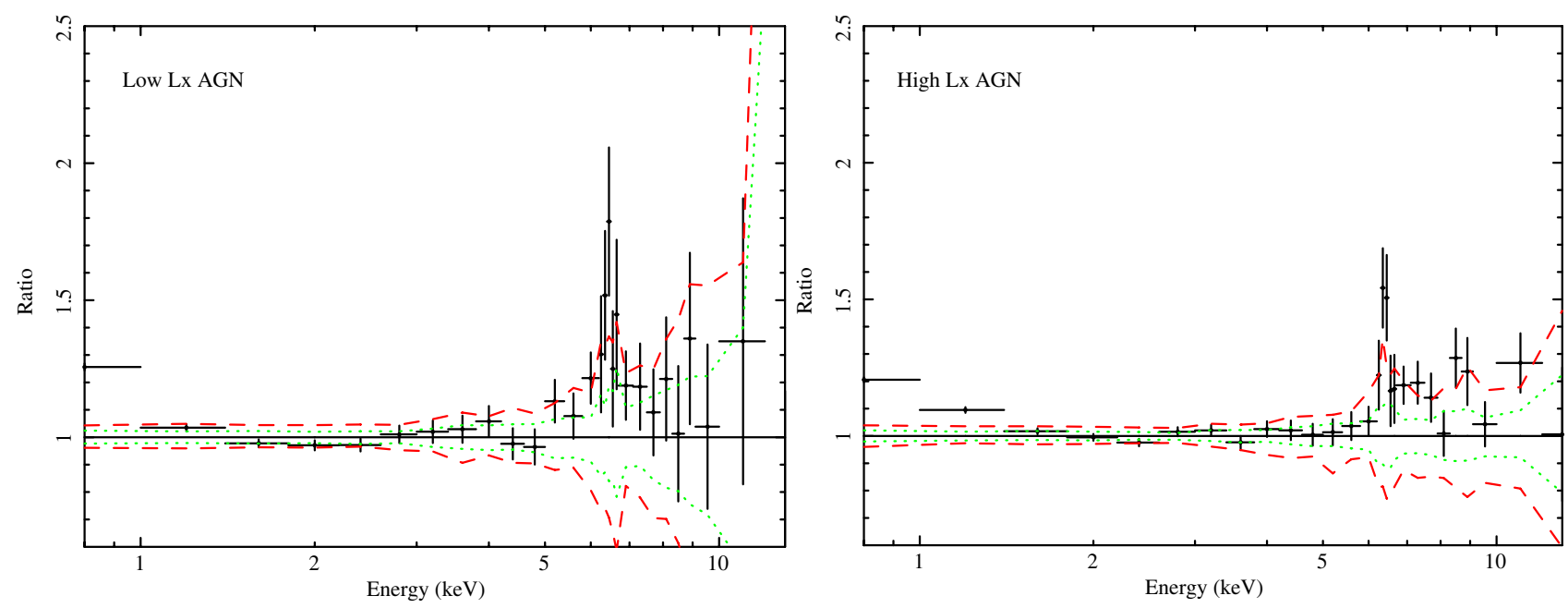

Fig. 13. Average spectrum to simulated continuum ratio corresponding to the luminosity subsamples. Error bars: real average spectrum, dashed lines: $2 \sigma$ limit, dotted lines: $1 \sigma$ limit.

used here is designed to study the 2 to $10 \mathrm{keV}$ rest-frame range to study the $\mathrm{Fe} \mathrm{K} \alpha$ line properties that minimize the contribution of the underlying continuum and observational effects. However, for highly absorbed sources $\left(N_{\mathrm{H}}>10^{23} \mathrm{~cm}^{-2}\right)$, the way the rescaling is carried out can give larger weights during the averaging process to the more absorbed sources. In the final average spectra, this produces a feature that could mimic the shape of a reflection component (Corral et al., in prep.). However, the number of highly absorbed sources is too small to be responsible for the whole amount of observed reflection. As a safety test, we removed the eight most absorbed $\operatorname{AGN}\left(N_{\mathrm{H}}>10^{23} \mathrm{~cm}^{-2}\right)$ from the average of absorbed AGN. Given that the remaining number of sources is small in this case, $R$ cannot be well constrained, but we obtain a lower limit of $R>1.1$ at the $90 \%$ confidence level. In summary, although the values obtained for the reflection components reported here have to be taken as tentative, the difference in the amount of reflection between absorbed and unabsorbed AGN seems to be real. For unabsorbed sources, our results excellently agree with those from studies of local AGN (Nandra et al. 2007), from the average of large samples of distant AGN (Chaudhary \& Brusa 2010) and with the predictions of theoretical models (Ballantyne 2010).

\subsection{Dependence on redshift and luminosity}

As we showed in Sect. 5, there seems to be a difference between the spectral shape for low- $\left(L_{\mathrm{x}}<10^{44} \mathrm{erg} \mathrm{s}^{-1}\right)$ and high- $\left(L_{\mathrm{x}}>10^{44} \mathrm{erg} \mathrm{s}^{-1}\right)$ luminosity type $1 \mathrm{AGN}$. To explore this possible difference, we constructed the average spectrum for both luminosity subsamples. The resulting ratios of the average spectra to the simulated continua are shown in Fig. 13. We find that the best-fit model for both samples consists in a power law with a narrow $\mathrm{Fe} \mathrm{K} \alpha$ line centered on $\sim 6.4 \mathrm{keV}$ and a reflection component. Consistently with the results reported in Sect. 5 we find the photon index of the average spectrum for the low-luminosity subsample to be (marginally) larger than the one for the high-luminosity subsample. The large error on $\Gamma$ in the low-luminosity subsample is likely caused by the larger dispersion of the photon index distribution (see Fig. 8). For the lowluminosity subsample, the line $E W$ seems to be higher, $E W=$ $110 \pm 30 \mathrm{eV}$, than for the high-luminosity subsample, $E W=$ $80 \pm 30 \mathrm{eV}$ (the so-called Iwasawa-Taniguchi effect, Iwasawa $\&$ Taniguchi 1993), but both values are consistent within errors.
The resulting reflection fraction also turns out to be marginally larger for the low-luminosity subsample, $R=0.8_{-0.5}^{+0.8}$, than for the high-luminosity sample, $R=0.3 \pm 0.1$, although it is not well constrained for the former sample. These results agree with models that predicts a decrease of the torus covering fraction as the luminosity increases (Lawrence 1991), thus decreasing the reprocessing of the radiation within the torus and also explaining the Iwasawa-Taniguchi effect. Evidence of this decrement of the covering fraction as a function of the luminosity have been reported in recent works (Maiolino et al. 2007; Della Ceca et al. 2008), which point out the need for the simplest unified schemes to be revised.

We also explored the possible dependence of the spectral shape on redshift. To this end, we again constructed average spectra by dividing the sample in different redshift bins. In this case we did not detect any significant trend of the resulting averaged spectral shape with redshift. Nevertheless, we point out again that our sample only reaches redshift $\sim 2$, and it is above this value where, for example, Mateos et al. (2010) found this dependence to become stronger.

\section{Discussion}

In the previous sections we discussed the possible existence of several statistical correlations. First, we found an anti-correlation between the photon index and the X-ray luminosity. This correlation is significant in the analysis of the single spectra, but it is also marginally present in the analysis of the average spectra. The lower significance in the latter case is likely caused by the high intrinsic dispersion in the photon index distribution. The second correlation, found in the analysis of the average spectra, is the dependence of the intensity of the reflection component with both the AGN "type" and luminosity. In particular, the reflection component seems to be stronger in absorbed AGN and in low-luminosity AGN.

An anti-correlation between the photon index and the X-ray luminosity has been recently reported by Green et al. (2009) and Young et al. (2009), whereas Saez et al. (2008) found a "positive" correlation. It has to be noted that Saez et al. (2008) took into account type 1 and type $2 \mathrm{AGN}$ at the same time, the latter being more numerous than the former, whereas we here only considered type 1 AGN. The physical explanation for this anticorrelation is still a matter of debate in the recent literature. 
Several authors (Shemmer et al. 2008; Risaliti et al. 2009; Grupe et al. 2010) have reported a correlation between the photon index and the Eddington ratio. This dependence could explain the correlations we find if the Eddington ratio was the actual driver of the luminosity/photon index anti-correlation. In that case, the low-luminosity subsample could be sampling a different AGN population or a mixture of very different accretion states, which could explain the higher dispersion found for the photon indices. We are currently studying this hypothesis in deeper detail.

The observed anti-correlation between the reflection component intensity and the luminosity found in the analysis of the average spectra confirms similar trends that were already observed in other samples (e.g. Nandra et al. 1997). If the observed reflection component is related to the molecular torus, the trend can be well explained in the context of the receding torus model according to which the molecular torus-covering fraction (and thus the intensity of the reflected component) decreases with the luminosity.

On the contrary, the difference seen in the observed reflection component between absorbed and unabsorbed AGN is, at the moment, troublesome because it is not easily reconciled with the unified model (which predicts a larger reflection component for unobscured sources, see e.g. Krolik et al. 1994; Murphy \& Yaqoob 2009). It is worth noting that similar results as those reported here (i.e. more reflection in absorbed objects) where obtained by Malizia et al. (2003) and by Deluit \& Courvoisier (2003) from the analysis of the average spectra of local type 1 and type 2 AGN observed with BeppoSAX; yet Burlon et al. (2011) have recently obtained the opposite trend from the analysis of the type 1 and type 2 AGN observed in the SWIFTBAT, although with large uncertainties. Further detailed studies on this particular aspect are clearly needed.

\section{Conclusions}

We have analyzed the X-ray spectra corresponding to all identified AGN within the XBS sample.

From the individual analysis and according to our fitting criteria, we find that

- Most AGN are well fitted by a simple unabsorbed power law model. The most common deviation from this shape are neutral intrinsic absorption and soft-excess emission.

- In agreement with the AGN unified model, most type 2 AGN are absorbed $\left(N_{\mathrm{H}}>4 \times 10^{21} \mathrm{~cm}^{-2}\right)$ and by larger amounts of intrinsic material than type 1 AGN, which are most unabsorbed. Nonetheless, deviations from this simple version of the unified model are found, and are more frequent in type 2 AGN.

- We find that the fraction of exceptions to the unified model is of $5 \%$ for the whole sample (only $3 \%$ if we take into account the errors on the measured intrinsic absorption). The fraction of type 1 AGN among absorbed sources is $17 \%$ and $31 \%$ for the HBSS and the BSS, respectively. The different values for the two samples are likely due to the larger number of unidentified sources within the BSS. The fraction of type 2 AGN among unabsorbed sources turns to be $3 \%$ and $1 \%$ for the HBSS and the BSS, respectively.

- We find that the X-ray spectral photon index for type 1 AGN is anti-correlated with the hard X-ray luminosity. When the type 1 AGN sample is split into high- and low-luminosity subsamples, we find that the intrinsic photon index for both samples is different almost at the $3 \sigma$ level in the plane photon index vs. intrinsic dispersion. We compute an average photon index and intrinsic dispersion of $\langle\Gamma\rangle=2.11 \pm 0.04$ $(2.00 \pm 0.05)$ and $\sigma=0.29 \pm 0.04(0.20 \pm 0.04)$ for the low(high) luminosity sample.

- We find that the so-called "soft-excess" is a common characteristic of AGN and it clearly displays different properties and origin for unabsorbed and absorbed AGN.

From the constructed average spectra we find that

- The average spectrum for type 2 AGN is different in the HBSS and the BSS samples as a result of a larger amount of absorbed sources in the HBSS. We do not find any significant differences between the type 1 AGN average spectra for these two samples.

- Apart from the amount of absorption, the differences between average spectra corresponding to absorbed and unabsorbed AGN are caused by an increase in the amount of reflection.

- We do not detect a significant relativistic Fe $\mathrm{K} \alpha$ emission line on the average spectrum for unabsorbed sources. We estimate an upper limit for a broad relativistic contribution to the line of $230 \mathrm{eV}$ at the $3 \sigma$ confidence level.

- When dividing the type 1 AGN sample into high- and lowluminosity sources, we find that the narrow $\mathrm{Fe} \mathrm{K} \alpha$ line $E W$ seems to decrease as the luminosity increases, which is consistent with the so-called Iwasawa-Taniguchi effect, although the resulting values for the high- and low-luminosity subsamples are consistent within errors $(E W=110 \pm 30$ and $80 \pm 30 \mathrm{eV}$ for the low- and high-luminosity subsamples, respectively). We find moreover that the amount of reflection may also decrease with luminosity, which supports models in which the covering fraction of the putative torus decreases as the intrinsic luminosity increases.

Acknowledgements. We would like to thank the referee for providing us with constructive comments and suggestions. We acknowledge financial support from ASI (grant n.I/088/06/0 and COFIS contract. It is a pleasure to thank Tommaso Maccacaro, Mike Watson and Valentina Braito for their initial efforts and contributions to the XBS project.

\section{References}

Akylas, A., \& Georgantopoulos, I. 2009, A\&A, 500, 999

Akylas, A., Georgakakis, A., \& Georgantopoulos, I. 2004, MNRAS, 353, 1015 Antonucci, R. 1993, ARA\&A, 31, 473

Arnaud, K. A. 1996, in Astronomical Data Analysis Software and Systems V, ed. G. H. Jacoby, \& J. Barnes, ASP Conf. Ser., 101, 17

Ballantyne, D. R. 2010, ApJ, 716, L27

Bassani, L., Dadina, M., Maiolino, R., et al. 1999, ApJS, 121, 473

Bianchi, S., Guainazzi, M., \& Chiaberge, M. 2006, A\&A, 448, 499

Bianchi, S., Bonilla, N. F., Guainazzi, M., Matt, G., \& Ponti, G. 2009a, A\&A, 501,915

Bianchi, S., Guainazzi, M., Matt, G., Fonseca Bonilla, N., \& Ponti, G. 2009b, A\&A, 495, 421

Brusa, M., Gilli, R., \& Comastri, A. 2005, ApJ, 621, L5

Burlon, D., Ajello, M., Greiner, J., et al. 2011, ApJ, 728, 58

Caccianiga, A., Severgnini, P., Braito, V., et al. 2004, A\&A, 416, 901

Caccianiga, A., Severgnini, P., Della Ceca, R., et al. 2007, A\&A, 470, 557

Caccianiga, A., Severgnini, P., Della Ceca, R., et al. 2008, A\&A, 477, 735

Calzetti, D., \& Heckman, T. M. 1999, ApJ, 519, 27

Cappi, M., Panessa, F., Bassani, L., et al. 2006, A\&A, 446, 459

Chaudhary, P., \& Brusa, M. 2010, in AIP Conf. Ser. 1248, ed. A. Comastri, L. Angelini, \& M. Cappi, 407

Corral, A., Page, M. J., Carrera, F. J., et al. 2008, A\&A, 492, 71

Crummy, J., Fabian, A. C., Gallo, L., \& Ross, R. R. 2006, MNRAS, 365, 1067

Dadina, M. 2008, A\&A, 485, 417

Della Ceca, R., Maccacaro, T., Caccianiga, A., et al. 2004, A\&A, 428, 383

Della Ceca, R., Caccianiga, A., Severgnini, P., et al. 2008, A\&A, 487, 119

Deluit, S., \& Courvoisier, T. 2003, A\&A, 399, 77 
Dickey, J. M., \& Lockman, F. J. 1990, ARA\&A, 28, 215

Elitzur, M., \& Shlosman, I. 2006, ApJ, 648, L101

Galbiati, E., Caccianiga, A., Maccacaro, T., et al. 2005, A\&A, 430, 927

Gilli, R., Comastri, A., \& Hasinger, G. 2007, A\&A, 463, 79

Green, P. J., Aldcroft, T. L., Richards, G. T., et al. 2009, ApJ, 690, 644

Grupe, D., Mathur, S., \& Komossa, S. 2004, AJ, 127, 3161

Grupe, D., Komossa, S., Leighly, K. M., \& Page, K. L. 2010, ApJS, 187, 64

Guainazzi, M., Bianchi, S., \& Dovčiak, M. 2006, Astron. Nachr., 327, 1032

Hickox, R. C., \& Markevitch, M. 2006, ApJ, 645, 95

Isobe, T., \& Feigelson, E. D. 1986, Bulletin d'Information du Centre de Données Stellaires, 31, 209

Isobe, T., \& Feigelson, E. D. 1990, in BAAS, 22, 917

Iwasawa, K., \& Taniguchi, Y. 1993, ApJ, 413, L15

Kawaguchi, T., Shimura, T., \& Mineshige, S. 2001, ApJ, 546, 966

Krolik, J. H., Madau, P., \& Zycki, P. T. 1994, ApJ, 420, L57

Laor, A. 1991, ApJ, 376, 90

Lawrence, A. 1991, MNRAS, 252, 586

Liedahl, D. A., Osterheld, A. L., \& Goldstein, W. H. 1995, ApJ, 438, L115

López-Santiago, J., Micela, G., Sciortino, S., et al. 2007, A\&A, 463, 165

Maccacaro, T., Gioia, I. M., Wolter, A., Zamorani, G., \& Stocke, J. T. 1988, ApJ, 326,680

Magdziarz, P., \& Zdziarski, A. A. 1995, MNRAS, 273, 837

Mainieri, V., Hasinger, G., Cappelluti, N., et al. 2007, ApJS, 172, 368

Maiolino, R., Shemmer, O., Imanishi, M., et al. 2007, A\&A, 468, 979

Malizia, A., Bassani, L., Stephen, J. B., et al. 2003, ApJ, 589, L17

Mateos, S., Barcons, X., Carrera, F. J., et al. 2005a, A\&A, 433, 855

Mateos, S., Barcons, X., Carrera, F. J., et al. 2005b, A\&A, 444, 79
Mateos, S., Carrera, F. J., Page, M. J., et al. 2010, A\&A, 510, A35

Mewe, R., Lemen, J. R., \& van den Oord, G. H. J. 1986, A\&AS, 65, 511

Middleton, M., Done, C., \& Gierliński, M. 2007, MNRAS, 381, 1426

Murphy, K. D., \& Yaqoob, T. 2009, MNRAS, 397, 1549

Nandra, K., George, I. M., Mushotzky, R. F., Turner, T. J., \& Yaqoob, T. 1997, ApJ, 477, 602

Nandra, K., O’Neill, P. M., George, I. M., \& Reeves, J. N. 2007, MNRAS, 382, 194

Nicastro, F. 2000, ApJ, 530, L65

Page, M. J., Loaring, N. S., Dwelly, T., et al. 2006, MNRAS, 369, 156 Panessa, F., \& Bassani, L. 2002, A\&A, 394, 435

Reeves, J. N., \& Turner, M. J. L. 2000, MNRAS, 316, 234

Reeves, J. N., Turner, M. J. L., Ohashi, T., \& Kii, T. 1997, MNRAS, 292, 468

Risaliti, G., Young, M., \& Elvis, M. 2009, ApJ, 700, L6

Ross, R. R., Fabian, A. C., \& Mineshige, S. 1992, MNRAS, 258, 189

Saez, C., Chartas, G., Brandt, W. N., et al. 2008, AJ, 135, 1505

Severgnini, P., Caccianiga, A., Braito, V., et al. 2003, A\&A, 406, 483

Shemmer, O., Brandt, W. N., Netzer, H., Maiolino, R., \& Kaspi, S. 2008, ApJ, 682,81

Shimura, T., \& Takahara, F. 1993, ApJ, 419, 78

Streblyanska, A., Hasinger, G., Finoguenov, A., et al. 2005, A\&A, 432, 395

Tozzi, P., Gilli, R., Mainieri, V., et al. 2006, A\&A, 451, 457

Treister, E., Urry, C. M., \& Virani, S. 2009, ApJ, 696, 110

Watson, M. G., Schröder, A. C., Fyfe, D., et al. 2009, A\&A, 493, 339

Worsley, M. A., Fabian, A. C., Bauer, F. E., et al. 2005, MNRAS, 357, 1281

Young, M., Elvis, M., \& Risaliti, G. 2009, ApJS, 183, 17 


\section{Appendix A: Notes on individual sources}

During the spectral fit, those sources which are not well fitted using a simple power law model can be usually well fitted by using different additional components. Here, we describe how we decided between the different models that are an acceptable fit for each source:

- Leaky model: all sources for which a leaky model was selected as our best-fit model share a common spectral shape: a power law shape at high energies that drops around $\sim 2-3 \mathrm{keV}$ and an additional soft component. These sources are four type 2 AGN (XBSJ021822.2050615, XBSJ033845.7-322253, XBSJ040758.9-712833 and XBSJ112026.7+431520) and two type 1 AGN (XBSJ091828.4+513931 and XBSJ095218.9-013643). XBSJ095218.9-013643 is a NLSy1 (narrow line Seyfert 1) whose intriguing X-ray spectral shape (a very steep photon index and large amount of absorption that partially covers the central source) and variability (variability of a factor of 4 in the soft X-rays) have been already studied in detail and presented in Grupe et al. (2004). In Fig. A.1 is shown an example of a leaky model fit. We find that leaving the soft photon index free to vary for all these sources does not significantly improve the fit. However, in all cases, this soft photon index steepens if it is left free to vary, which suggests the contribution of an additional soft component, most likely a thermal component given the low luminosity observed for this sources. In no case adding a thermal component to the leaky model significantly improves the fit, and by fitting a simple absorbed power law plus a thermal component always gives worse residuals at low energies than the leaky model.

- Warm absorption: we find that an additional ionized absorber gives a best fit in five cases (XBSJ030641.0283559, XBSJ052543.6-334856, XBSJ140100.0-110942, XBSJ140127.7+025605, XBSJ223547.9-255836, all type 1 AGN), although the ionized absorber parameters, mainly the ionization state of the absorber, are not well constrained in all cases. We selected this model as our best-fit model when the power-law residuals at low energies showed some evidence of an structured shape resembling absorption lines or edges. In two cases, the source also displays a soft-excess (XBSJ030641.0-283559 and XBSJ223547.9-255836). One example of this model is again shown in Fig. A.1. The ionized absorber was added to the neutral one because of the way the spectral fit is carried out, i.e., our base-line model is a simple power law including neutral intrinsic absorption. It is worth noting, however, that none of the sources for which the best-fit model includes warm absorption need significant additional cold absorption, as can be seen in Table 7 .

- Absorption edges: in three cases (XBSJ100926.5+533426, XBSJ102412.3+042023 and XBSJ204159.2-321439), an absorption edge has to be added to the simple power law model to obtain an acceptable fit. It is not clear whether these edges are real or an instrumental effect given the energies at which they are found, but they could be caused by a warm absorber that our simple Xspec absori model is not able to fit properly. See again Fig. A.1.

- Reflection component: we find that a simple power law plus a neutral reflection component is a good fit in four cases (XBSJ031311.7-765428, XBSJ043448.3-775329, XBSJ052108.5-251913 and XBSJ101922.6+412049; all type 1 AGN). We use neutral reflection in all cases (pexrav model in Xspec) since our intention is not to determine where this reflection component originates in. Given the data quality, we can only estimate the amount of reflection by the reflection fraction $R$ in the pexrav model. Nevertheless, we find that in all cases but one (XBSJ031311.7-765428) the reflection component is most likely coming from Compton-thick material far away from the central source, the putative torus in unified models, given the spectral shape, a rather flat continuum at high energies, and the characteristics of a possible $\mathrm{Fe} \mathrm{K} \alpha$ line. Note also that all but XBSJ043448.3-775329 show a soft-excess.

- Black body: a phenomenological black body component is needed to obtain an acceptable fit in 13 cases (XBSJ000532.7+200716, XBSJ005031.1520012, XBSJ015957.5+003309, XBSJ021808.3-045845, XBSJ023530.2-523045， XBSJ023713.5-522734， XBSJ 031851.9-441815, XBSJ065839.5-560813, XBSJ074312.1+ 742937, XBSJ141736.3+523028, XBSJ 153456.1+013033, XBSJ225118.0-175951 and XBSJ231601.7-424038; all type 1 AGN), all showing soft-excess. The physical origin for this soft component is not clear although a host galaxy thermal contribution is ruled out given its high luminosity in all cases but XBSJ000532.7+200716. In that case the low luminosity found for the black body component $(\sim 4 \times$ $10^{42} \mathrm{erg} \mathrm{s}^{-1}$ in the $0.5-2.0 \mathrm{keV}$ energy range) could be caused by thermal emission, but adding an Xspec mekal component does not improve the simple power law fit. More complex models recently proposed in the literature (Crummy et al. 2006; Middleton et al. 2007) cannot be used in our case given the data quality, and in any case, they are indistinguishable in the EPIC-covered energy range. In some cases, the need for a black body component instead of a more physically motivated model, could be just due to the data quality. For example, in seven cases, XBSJ005031.1520012, XBSJ015957.5+003309, XBSJ021808.3045845, XBSJ065839.5-560813, XBSJ074312.1+742937, XBSJ153456.1+013033 and XBSJ225118.0-175951 (see Table 7), an ionized absorber is also a good fit, but gives worse residuals that the black body model. This could be because of to both the data quality and the need of a better representation of the ionized absorber. And for XBSJ021808.3-045845, XBSJ023713.5522734, XBSJ074312.1+742937, XBSJ141736.3+523028 and XBSJ231601.7-424038 (see Table 8, note that XBSJ021828.3-045845 and XBSJ074312.1+742937 can be also fitted by using ionized absorption), the addition of a reflection component instead of a black body also significantly improves the fit. For the first two cases, this reflection component could derive from ionized material.

- Sources for which no best fit is found: we are unable to find an acceptable fit in 11 cases. We do not find that these sources share any common characteristic, and that an acceptable fit is not found could be simply due to our selection criteria based on the resulting null hypothesis probability. The simple power law fit for these sources is shown in Fig. A.2. In the case of XBSJ021822.2-050615 and XBSJ153456.1+013033, the fit corresponds to a leaky model and a power law plus a black body, which significantly improve the fit, but not enough to obtain a probability $>10 \%$. 

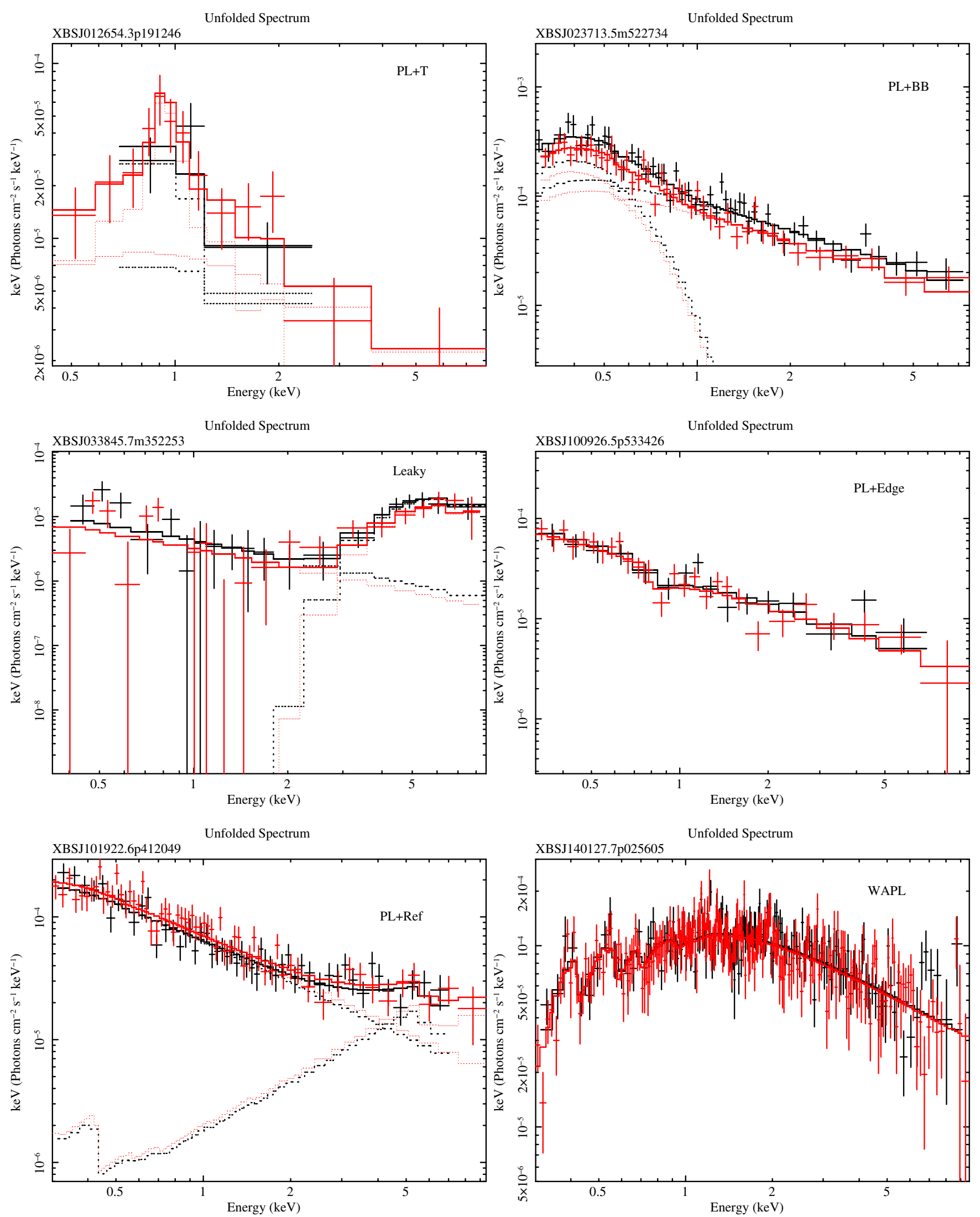

Fig. A.1. Unfolded spectra corresponding to the different additional components used during the spectral fit. From top to bottom and left to right: power law plus thermal component, power law plus black body, leaky model, power law and absorption edge, power law plus reflection component and power law and ionized absorber. 
A. Corral et al.: The X-ray spectral properties of the AGN population in the XMM-Newton bright serendipitous survey
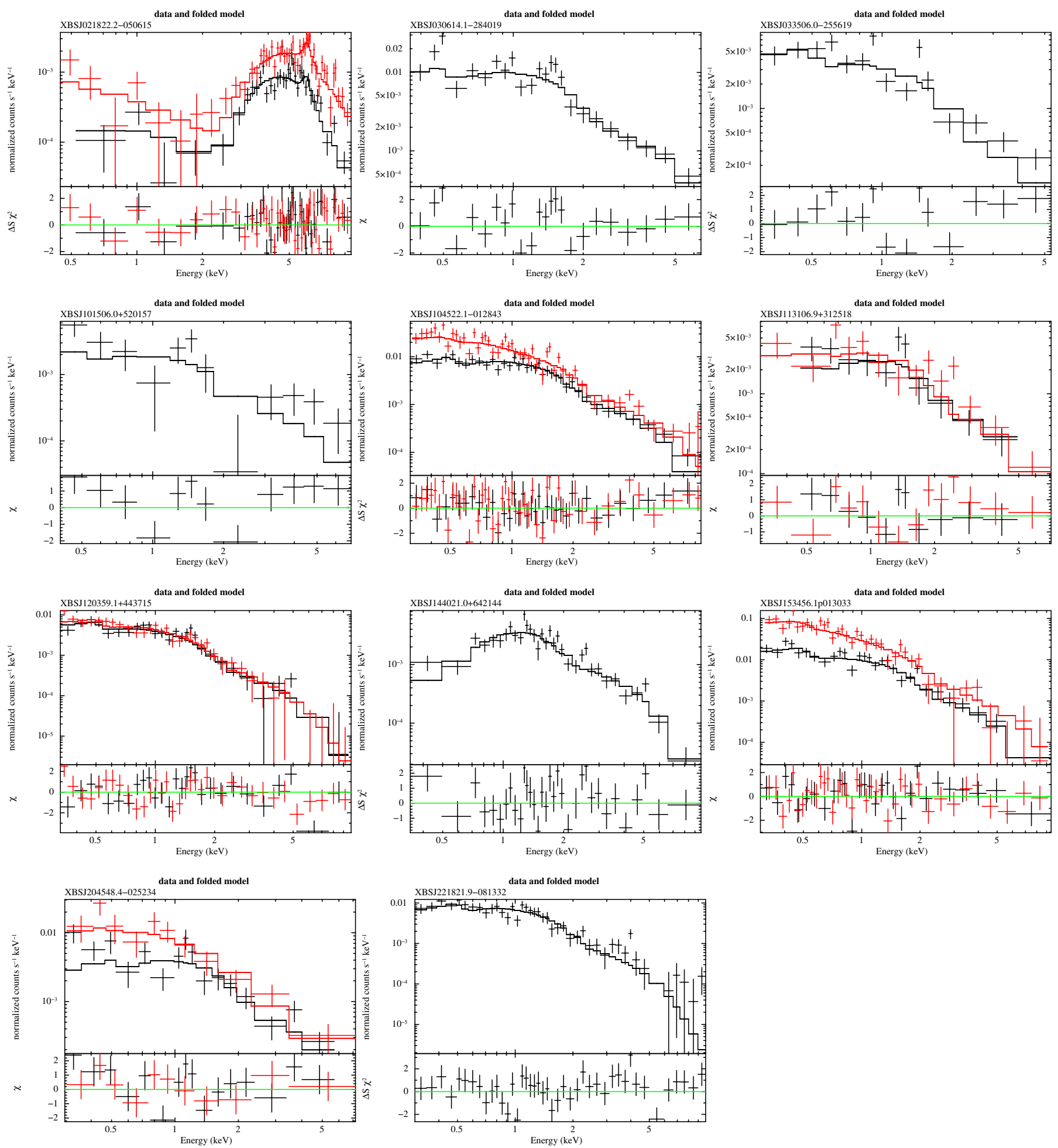

Fig. A.2. Data and residuals corresponding to the eleven sources for which no acceptable fit is found. 


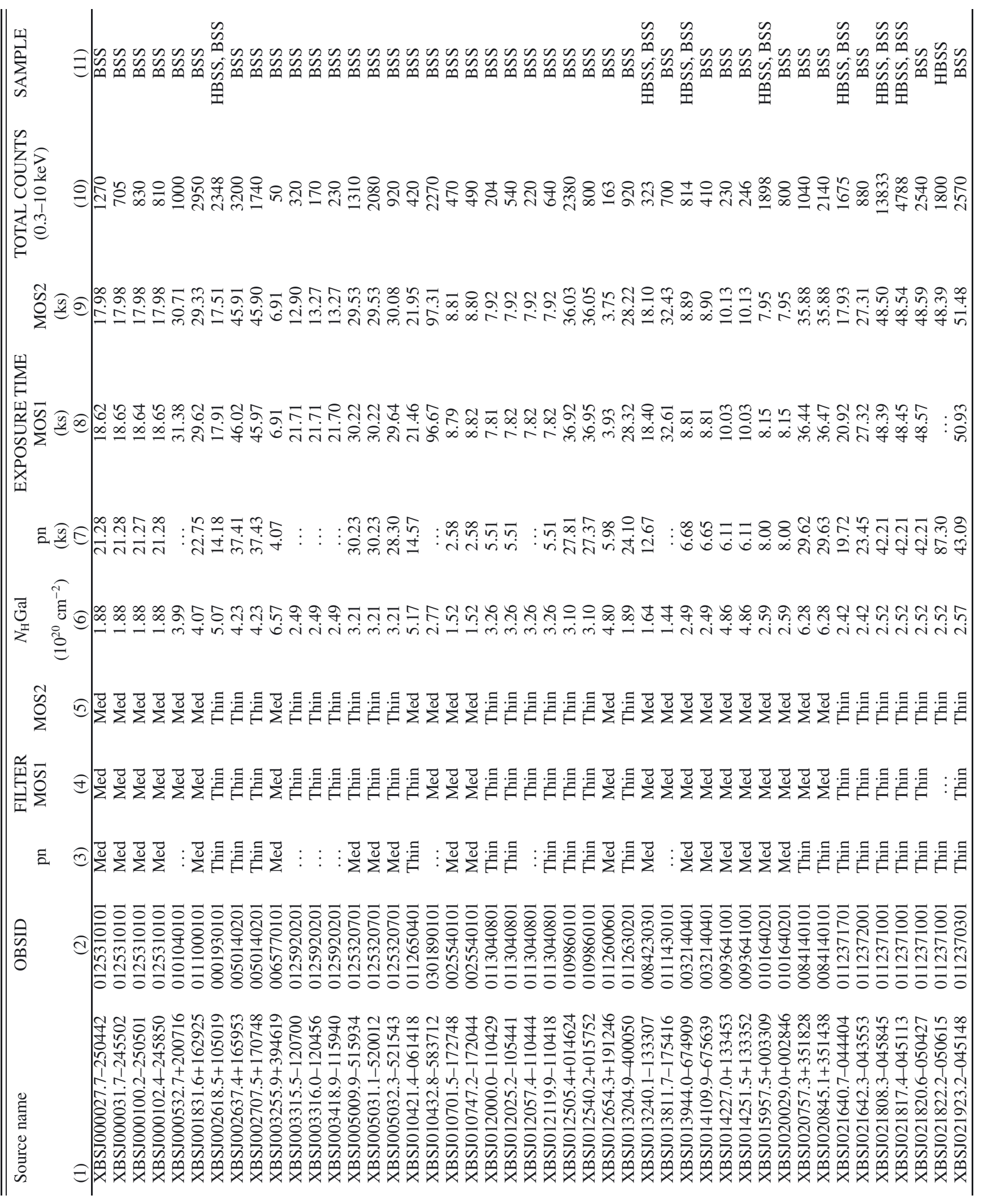


A. Corral et al.: The X-ray spectral properties of the AGN population in the XMM-Newton bright serendipitous survey

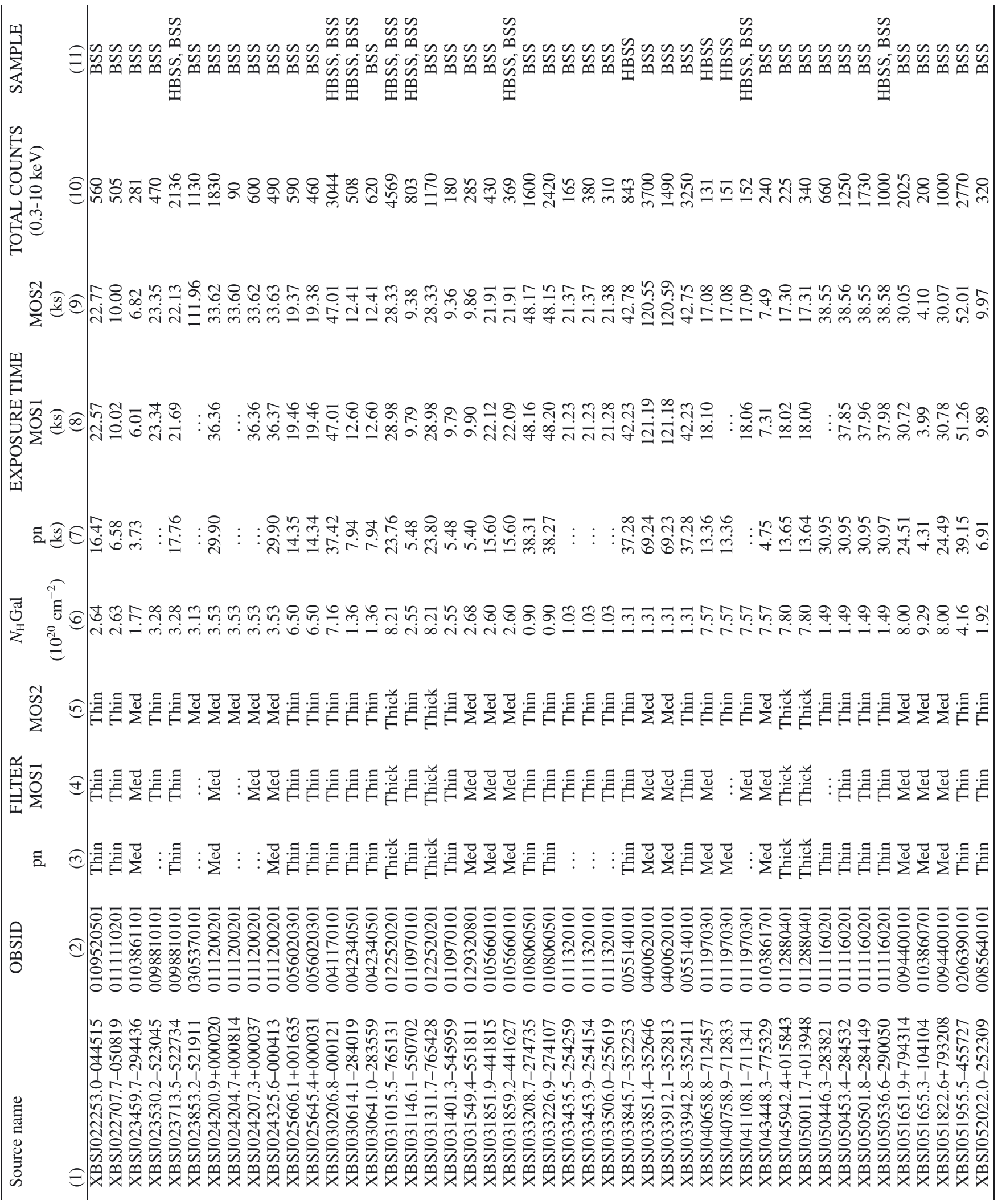




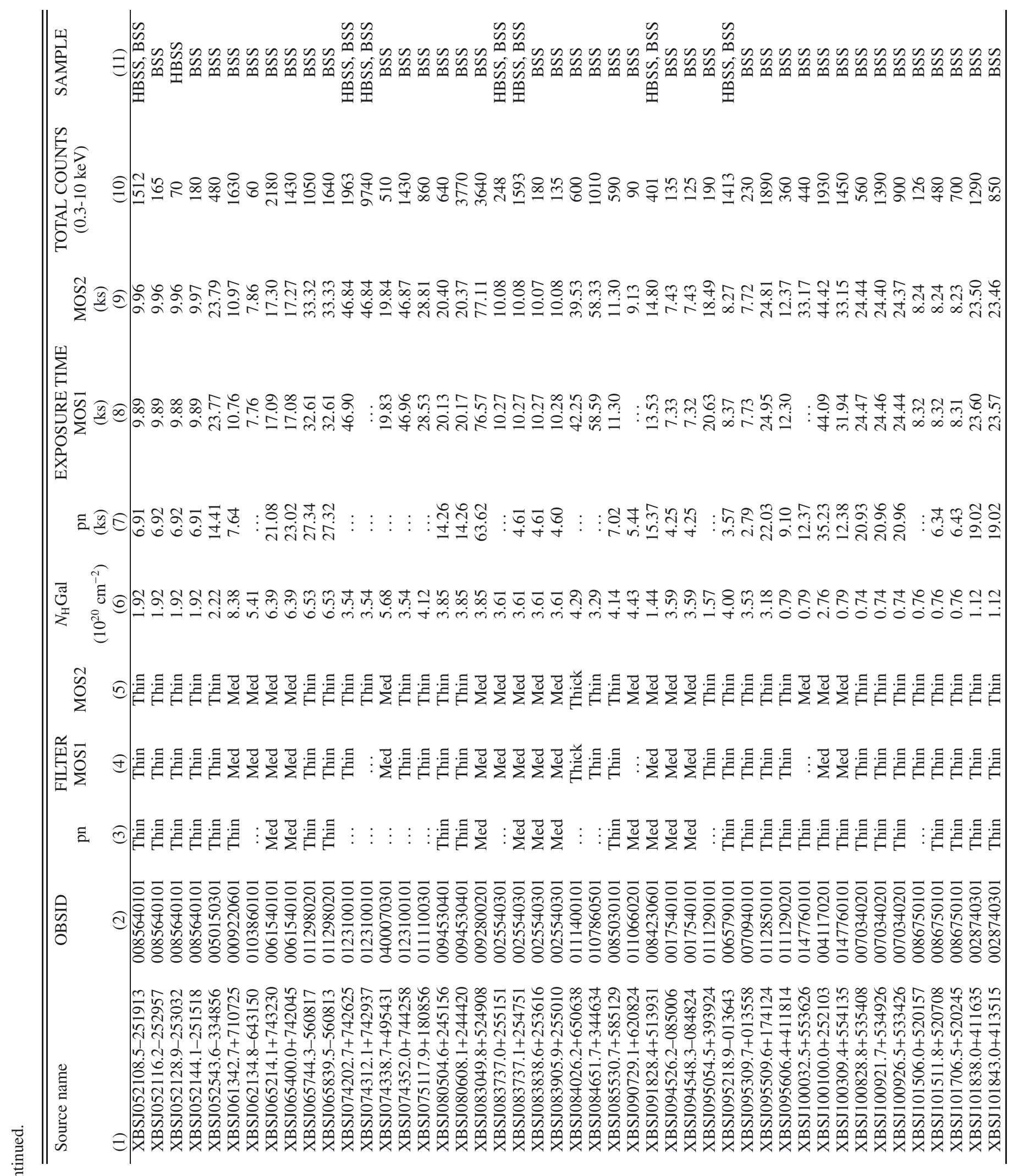


A. Corral et al.: The X-ray spectral properties of the AGN population in the XMM-Newton bright serendipitous survey

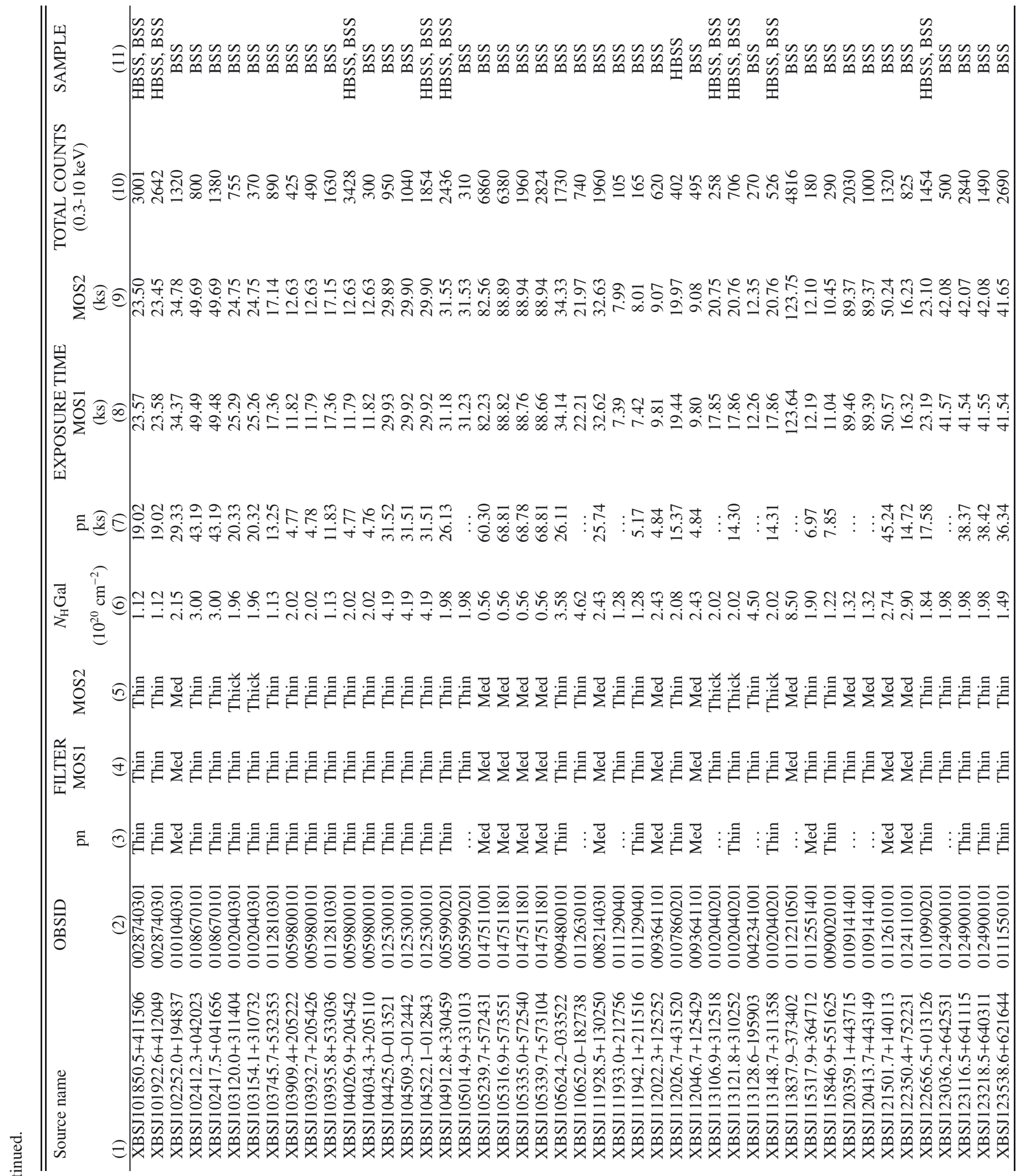




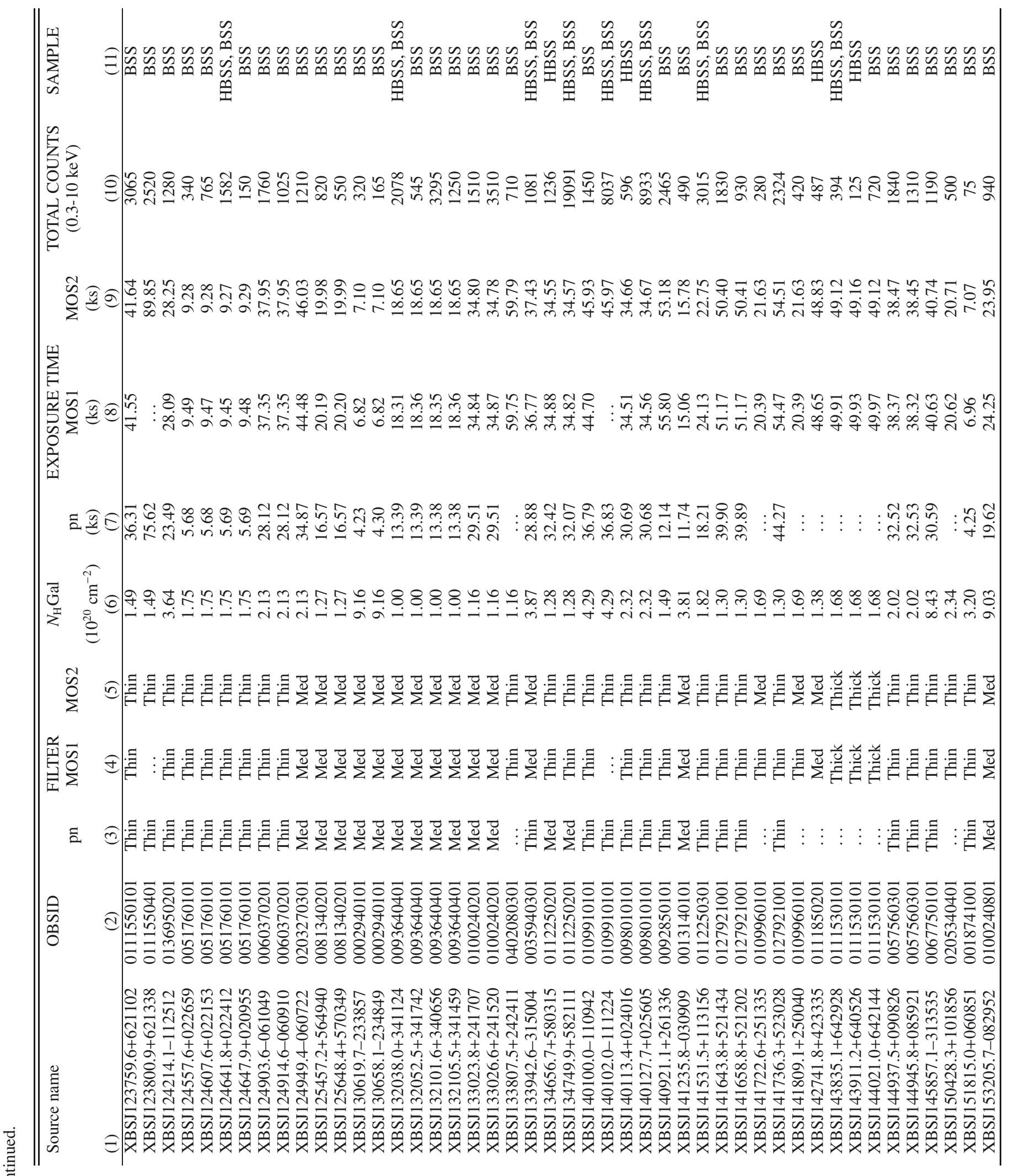


A. Corral et al.: The X-ray spectral properties of the AGN population in the XMM-Newton bright serendipitous survey

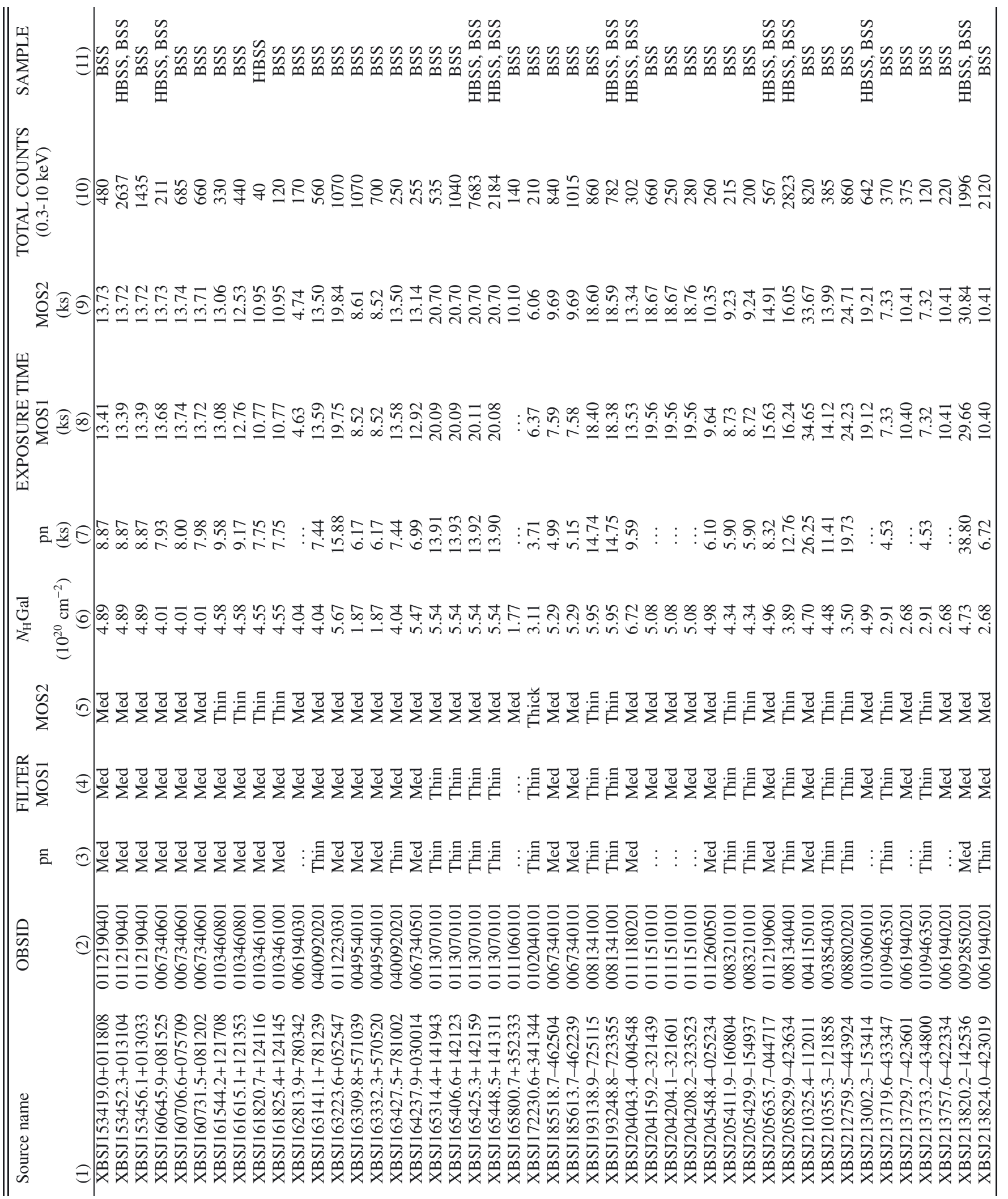




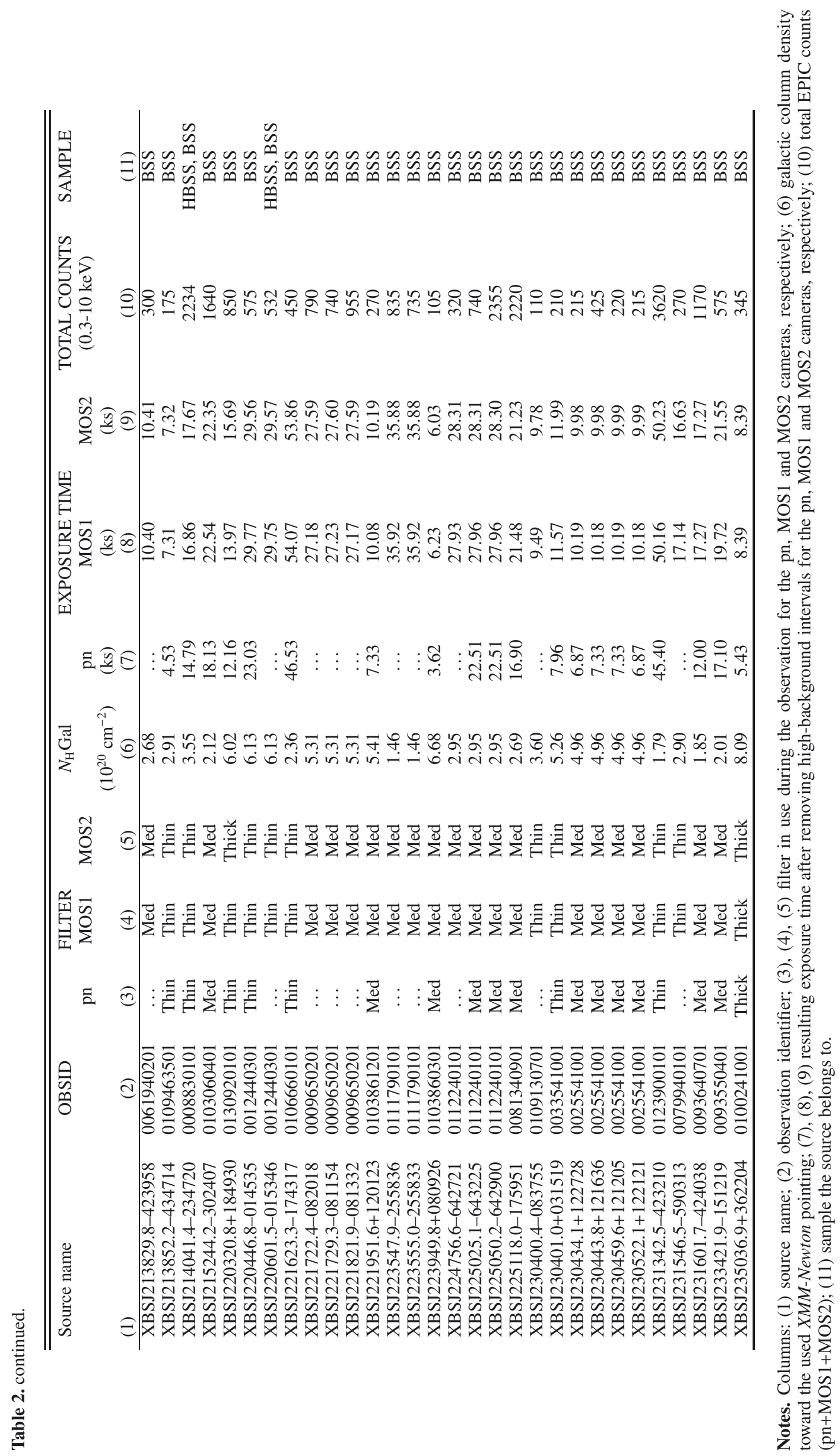


A. Corral et al.: The X-ray spectral properties of the AGN population in the XMM-Newton bright serendipitous survey

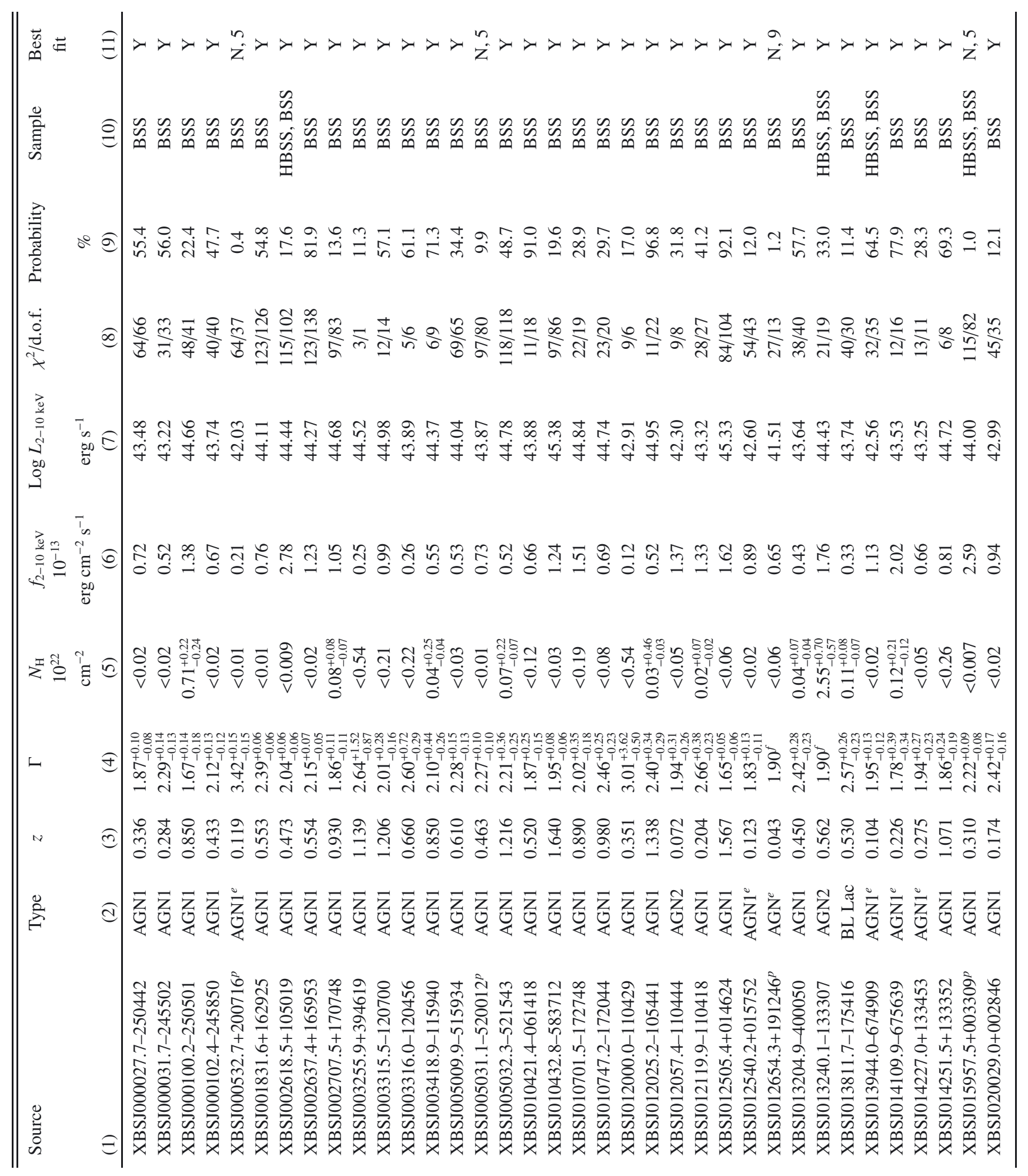




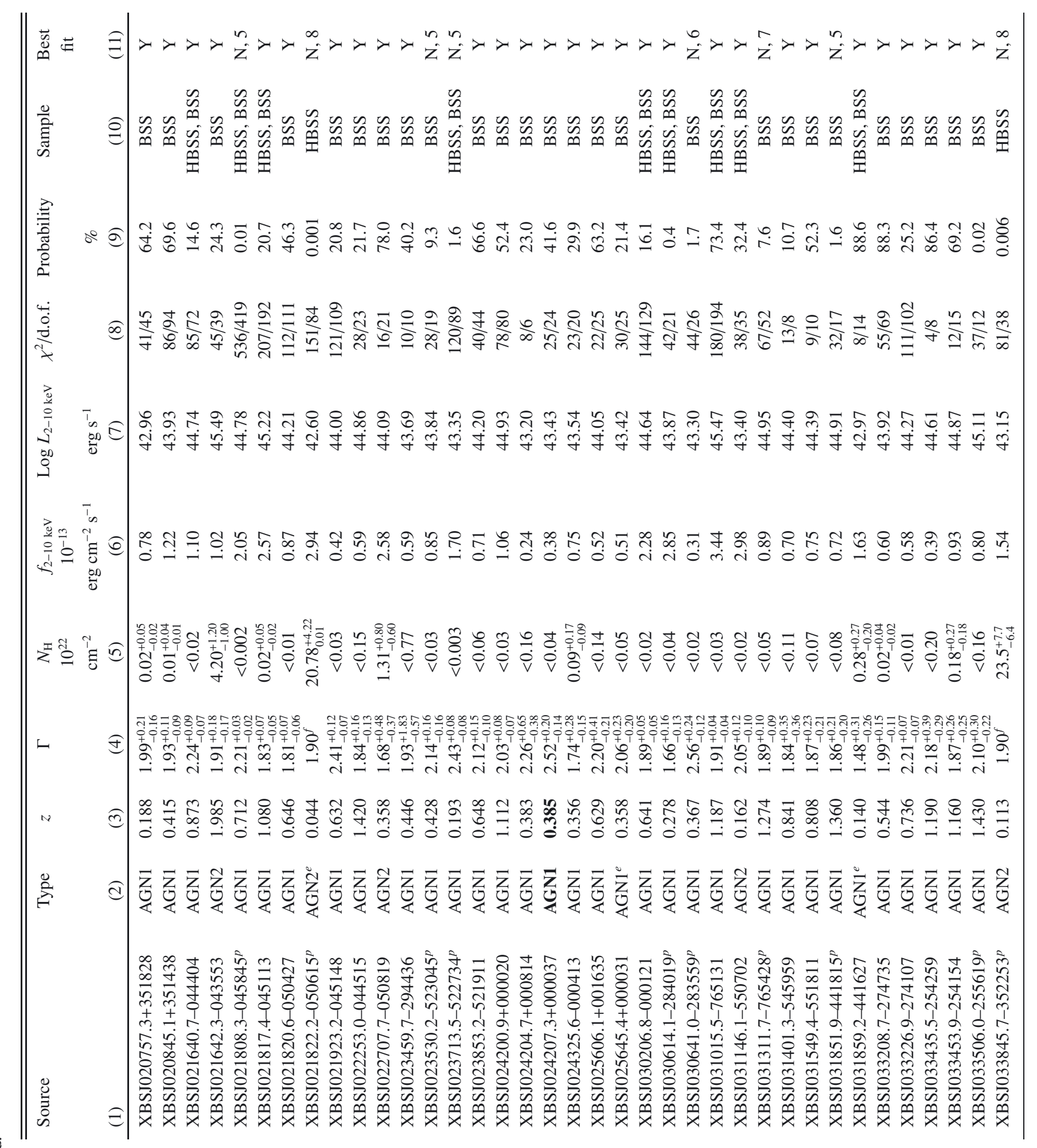


A. Corral et al.: The X-ray spectral properties of the AGN population in the XMM-Newton bright serendipitous survey

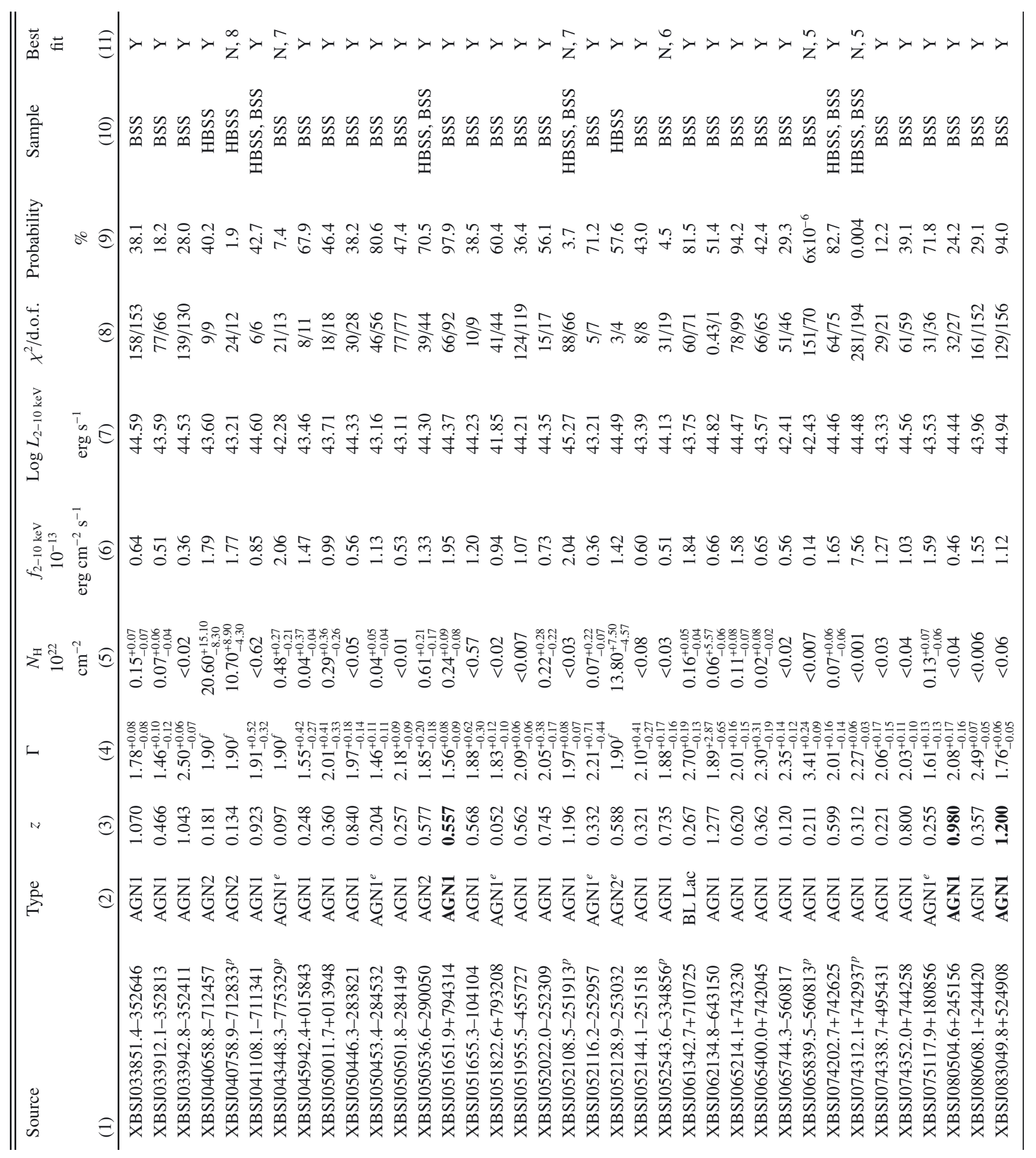




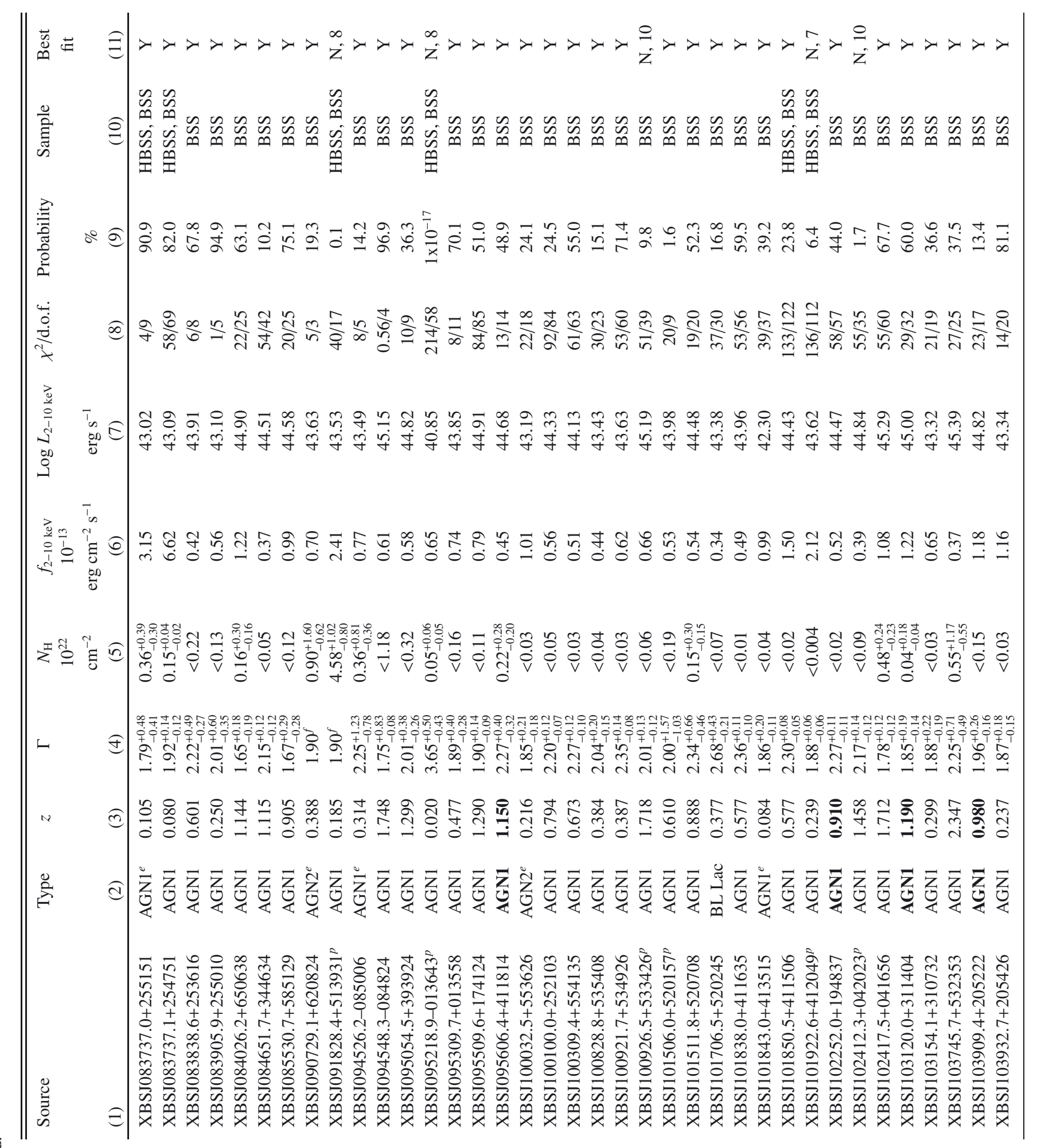


A. Corral et al.: The X-ray spectral properties of the AGN population in the XMM-Newton bright serendipitous survey

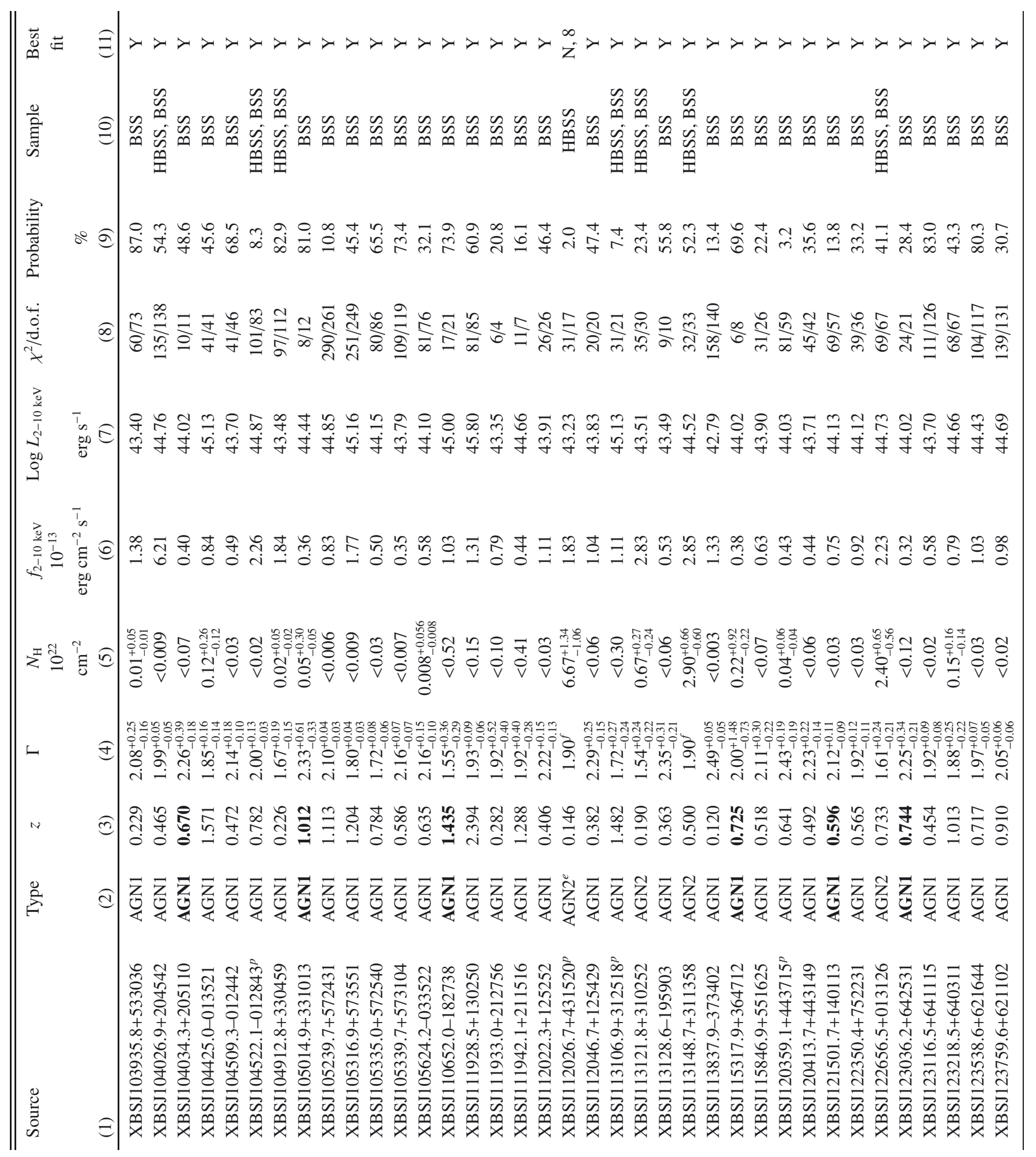




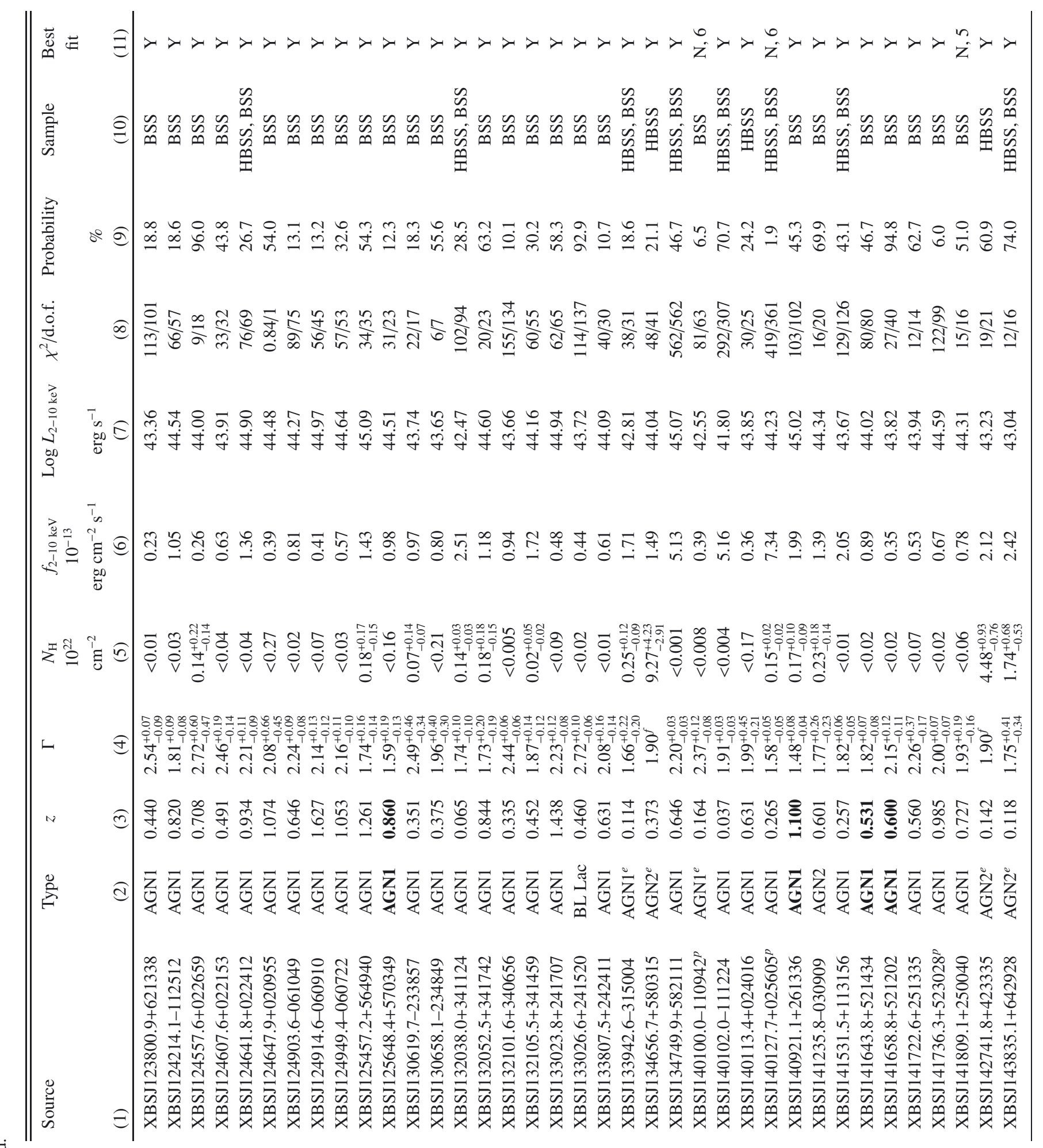


A. Corral et al.: The X-ray spectral properties of the AGN population in the XMM-Newton bright serendipitous survey

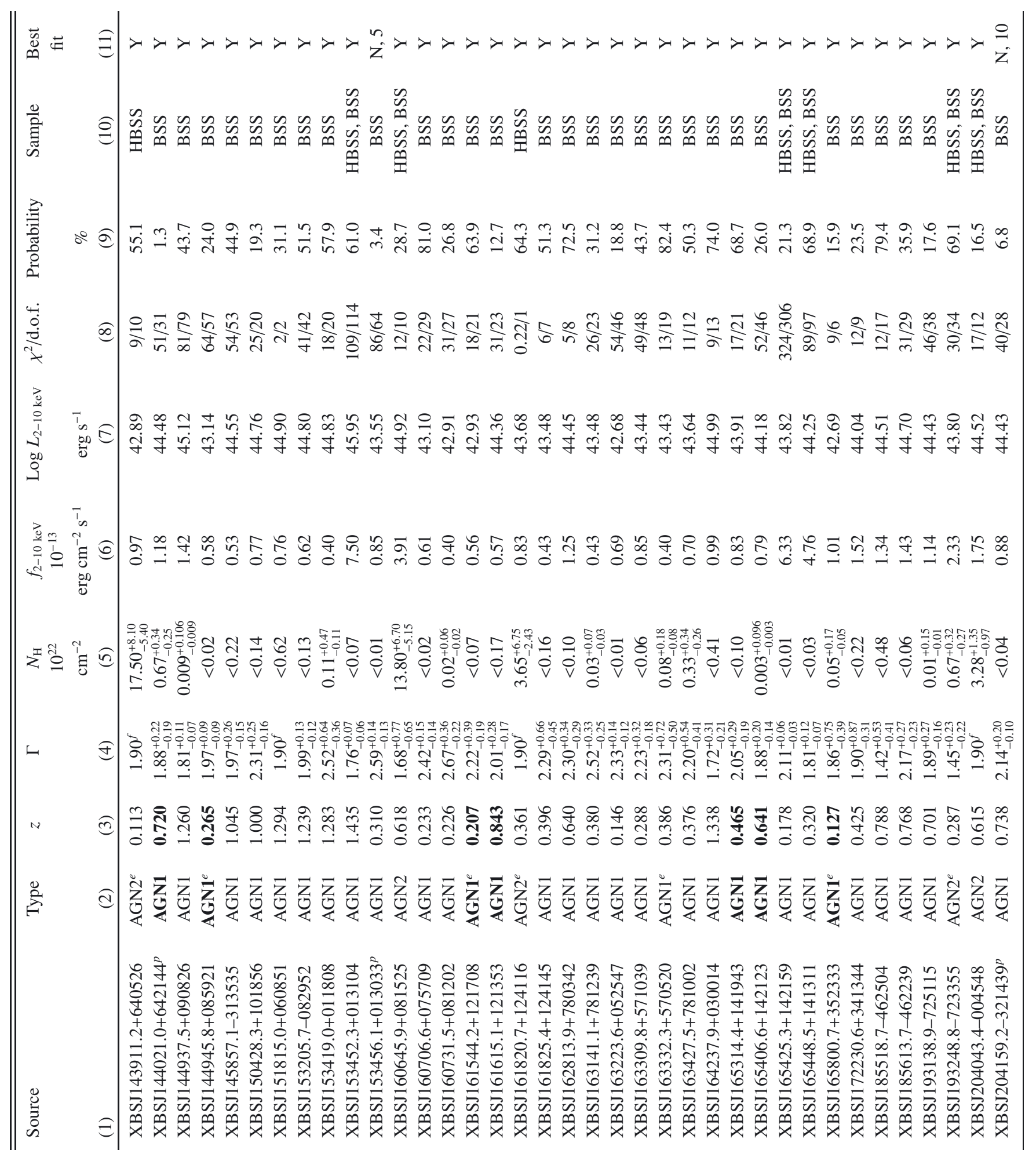




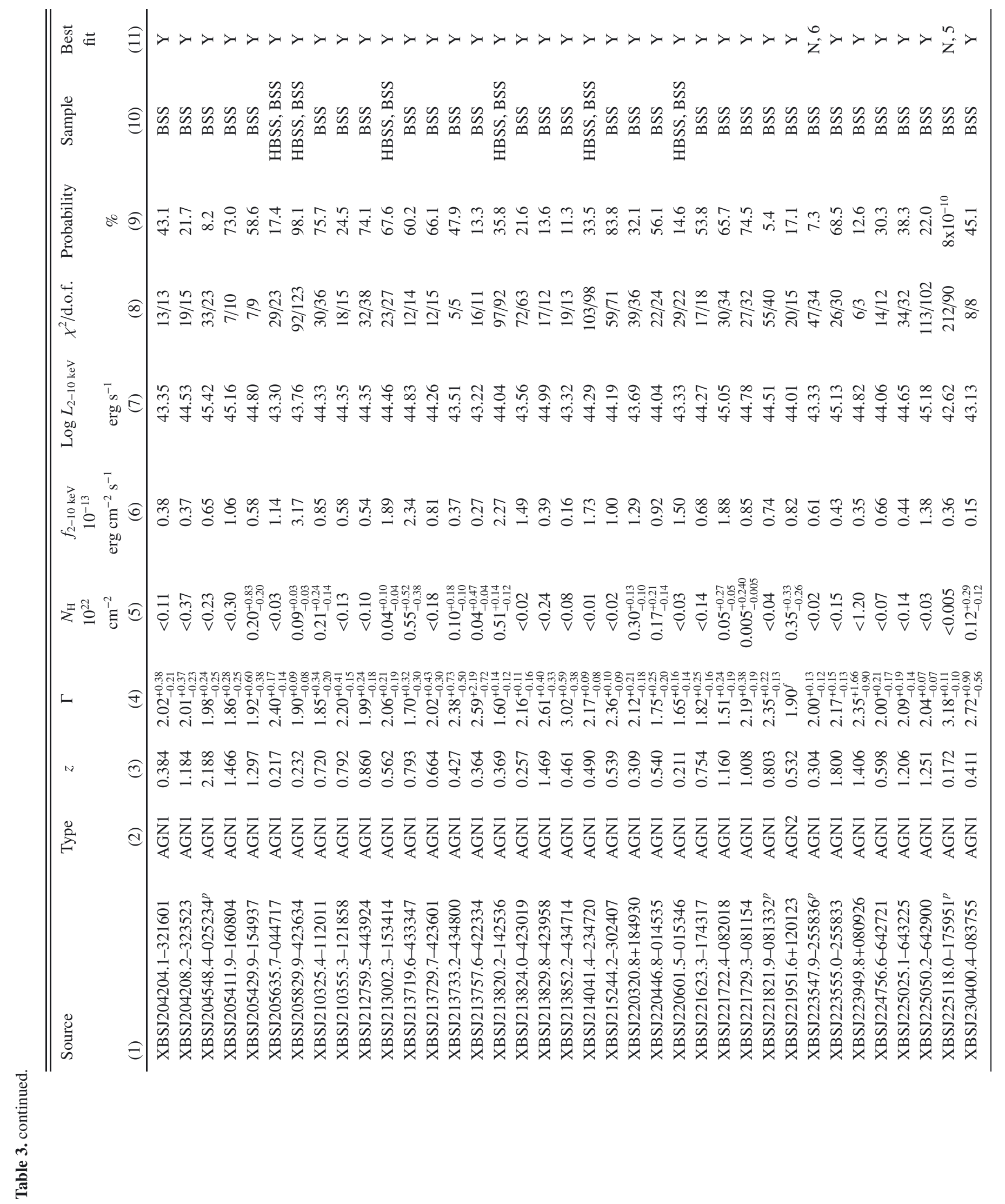

A42, page 38 of 39 
A. Corral et al.: The X-ray spectral properties of the AGN population in the XMM-Newton bright serendipitous survey

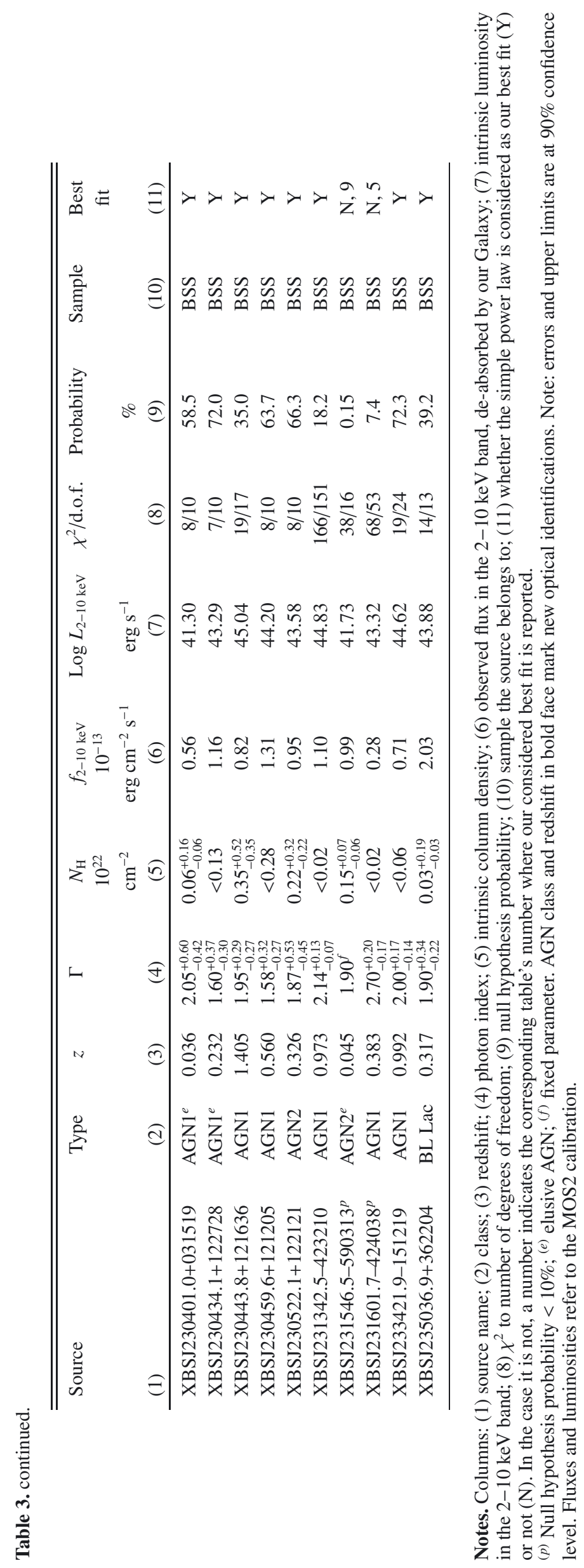

